\title{
Mixed Convective-Radiative Dissipative Magnetized Micropolar Nanofluid Flow over a Stretching Surface in Porous Media with Double Stratification and Chemical Reaction Effects: ADM-Padé Computation
}

\author{
P. K. Pattnaik, ${ }^{1}$ M. M. Bhatti $\mathbb{D}^{2},{ }^{2}$ S. R. Mishra, ${ }^{3}$ Munawwar Ali Abbas, ${ }^{4}$ and O. Anwar Bég ${ }^{5}$ \\ ${ }^{1}$ Department of Mathematics, Odisha University of Technology and Research, Bhubaneswar, Odisha 751029, India \\ ${ }^{2}$ College of Mathematics and Systems Science, Shandong University of Science and Technology, Qingdao, \\ Shandong 266590, China \\ ${ }^{3}$ Department of Mathematics, Siksha "O" Anusandhan University, Bhubaneswar, Odisha 751030, India \\ ${ }^{4}$ Department of Mathematics, University of Baltistan Skardu, Gilgit-Baltistan 16100, Pakistan \\ ${ }^{5}$ Multi-Physical Engineering Sciences Group, Mechanical Engineering, University of Salford, Salford M5 4WT, UK \\ Correspondence should be addressed to M. M. Bhatti; mmbhatti@sdust.edu.cn
}

Received 27 November 2021; Revised 18 January 2022; Accepted 25 January 2022; Published 14 February 2022

Academic Editor: Nauman Raza

Copyright ( 2022 P. K. Pattnaik et al. This is an open access article distributed under the Creative Commons Attribution License, which permits unrestricted use, distribution, and reproduction in any medium, provided the original work is properly cited.

\begin{abstract}
The present study deals with the electrically conducting micropolar nanofluid flow from a vertical stretching surface adjacent to a porous medium under a transverse magnetic field. Eringen's micropolar model is deployed for non-Newtonian characteristics and the Buongiorno nanofluid model employed for nanoscale effects (thermophoresis and Brownian motion). The model includes double stratification (thermal and solutal) and also chemical reaction effects, heat source, and viscous dissipation. Darcy's model is employed for the porous medium and a Rosseland diffusion flux approximation for nonlinear thermal radiation. The nonlinear governing partial differential conservation equations are rendered into nonlinear ordinary differential equations via relevant transformations. An innovative semi-numerical methodology combining the Adomian decomposition method (ADM) with Padé approximants and known as ADM-Padé is deployed to solve the emerging nonlinear ordinary differential boundary value problem with appropriate wall and free stream conditions in MATLAB software. A detailed parametric study of the influence of key parameters on stream function, velocity, microrotation (angular velocity), temperature, and nanoparticle concentration profiles is conducted. Furthermore, skin friction coefficient, wall couple stress coefficient, Nusselt number, and Sherwood number are displayed in tables. The validation of both numerical techniques used, i.e., ADM and ADM-Padé, against a conventional numerical $4^{\text {th }}$ order Runge-Kutta method is also included and significant acceleration in convergence of solutions achieved with the ADM-Padé approach. The flow is decelerated with greater buoyancy ratio parameter whereas microrotation (angular velocity) is enhanced. Increasing thermal and solutal stratification suppresses microrotation. Concentration magnitudes are boosted with greater chemical reaction parameter and Lewis number. Temperatures are significantly enhanced with radiative parameter. Increasing Brownian motion parameter depletes concentration values. The study finds applications in thermomagnetic coating processes involving nanomaterials with microstructural characteristics.
\end{abstract}

\section{Introduction}

The study of boundary layer flow and heat transfer in a mixture of fluids flowing across a continuous extending surface has numerous industrial applications, including thermal polymeric coating flows, surface deposition and finishing, enrobing, and mechanical handling equipment such as conveyors [1,2]. Crane [3] was among the first researchers to study the viscous Newtonian boundary layer equations for two-dimensional continuous flow across a flat, incompressible elastic sheet traveling in a straight line. He established a good foundation for subsequent studies involving multiple thermal and electromagnetic effects. Mishra et al. [4] recently investigated heat and mass transfer in magnetized viscoelastic fluid 
with conducting of a viscoelastic (Walters B') fluid on a stretching surface. This study also included chemical reaction and non-uniform heat source effects, which are commonly encountered in materials processing.

Mass transfer is a common occurrence in a wide variety of industrial technologies including metallurgical processing, food manufacture, and electromagnetic (smart) coating dynamics. It may involve multiple species diffusing through a fluid and is often accompanied with heat transfer. Whereas Fick's law is usually deployed for species diffusion, for thermal conduction the Fourier law is required. Due to the diverse applications in chemical, nuclear, and mechanical process and materials fabrication systems (extrusion, coating, enrobing, etc.), mathematical models of the combined effect of mass transfer and chemical reaction both with and without electromagnetic fields have stimulated considerable interest among engineers and scientists $[5,6]$. In high temperature applications, radiative heat transfer is also encountered (in addition to thermal conduction and thermal convection) and usually an algebraic flux model is employed in simulations to compute the effect of radiative flux on transport phenomena. Numerous authors [7-9] investigated the radiative convective heat and mass transport in magnetohydrodynamic (MHD) flows. Pattnaik et al. [10] investigated the unsteady magnetohydrodynamics free convection flow, heat, and mass transfer with variable temperature and concentration from an inclined surface embedded in a saturated constant permeability porous medium. They observed that the transport characteristics and substantially modified with heat source, inclination, porous drag force, and magnetic body force effects. Pattnaik et al. [11] also computed the influence of heat flux caused by a concentration and temperature gradient on an unsteady hydromagnetic flow from an infinite vertical porous plat, noting that the temperature and velocity are significantly altered by the magnetic field strength. In [12], it was noted that chemical reaction, heat source, and suction/injection at the wedge wall all have a significant effect on MHD boundary layer flow.

In many materials processing operations, non-Newtonian (rheological) fluids are utilized. These exhibit characteristics which deviate significantly from viscous Newtonian fluid models. Many non-Newtonian models are available including viscoplastic (Bingham), viscoelastic (Maxwell, Oldroyd-B), shear thinning pseudoplastic (Carreau, Cross, etc.). However, these models merely modify the shear stress tensor and do not include microstructural effects. The particles suspended in liquids deployed in many materials processing operations can spin; i.e., these fluids have a microstructure. Such effects cannot be simulated with standard non-Newtonian models. Therefore, a new branch of fluid dynamics, micromorphic fluid mechanics, was introduced by Eringen in the 1960s [13] which successfully simulates the angular velocity or microrotation of particles and molecules in non-Newtonian fluids. A simplification of the general micromorphic fluid model is the micropolar model [14] which restricts the degrees of freedom of the particles and also neglects axial contraction/ extension of microelements. This model and its modification to include thermal effects (thermo-micropolar model [15]) has proved immensely popular and has been deployed in many excellent studies since many different colloidal suspensions, smart fluids, and biological fluent media (e.g., blood, synovial fluid) can be accurately simulated by micropolar theory. A further advantage of micropolar fluid theory is that, by neglecting the spin (gyratory motion of microelements), the classical Navier-Stokes Newtonian model can be retrieved. Motivated by coating applications, many researchers have analyzed the external boundary layer flows of micropolar fluids from different geometries. These investigations have usually deployed advanced numerical methods since the boundary value problems investigated are highly nonlinear. Bhargava and Takhar [16] used a variational finite element method to compute the stagnation micropolar heat transfer characteristics on a translating surface. A number of magnetohydrodynamics coating flows of micropolar fluids have also been studied in recent years. Ezzat et al. [17] studied MHD micropolar coating heat transfer with thermal relaxation effects using Laplace transform and numerical approaches. Many other interesting applications of magnetic coating flows have been reviewed by Douglas [18] including microstructural effects, for which the micropolar fluid model is very appropriate. Mishra et al. [19] have further examined the effects of double stratification, i.e., thermal and solutal stratification in magneto-convective flows of micropolar fluids with heat generation and buoyancy effects. All these studies have confirmed the substantial modification in thermal, species, and flow characteristics computed with micropolar models compared with either Newtonian or simpler non-Newtonian models.

Nowadays, conventional fluids are being progressively superseded in favor of nanofluids due to their superior thermal properties. Choi and Eastman [20] introduced nanofluids as colloidal suspensions of base fluids containing nanoparticles. Base fluids include oils, polymers, water, etc. Nanoparticles include metallics and non-metal oxides, carbides, and nitrates. Popular nanoscale models used for simulating nanofluid transport characteristics are the Tiwari-Das model and the Buongiorno two-component nanofluid model. The latter features a species concentration equation for nanoparticles and also emphasizes thermophoretic body force and Brownian motion dynamics effects. Wakif et al. [21] developed a generalized Buongiorno nanofluid model with the purpose of analyzing the effect of magnetic field on hydrodynamic stability. Nanofluids improve the thermal conductivity of lowconducting fluids such as ethylene glycol, engine oil, or water. Nanofluids therefore achieve enhanced heat conductivities which makes them more favourable for engineering and technological processes than conventional base fluids. Nanofluids have been employed extensively in a wide variety of technical applications, including heat exchangers, thermal engineering, cancer therapy, propulsion, tribology, and biomedicine. Additionally, there are several applications of magnetic fields in physics, medicine, and engineering. In magnetic materials processing, as noted earlier, external magnetic fields can be deployed to regulate flow characteristics and also manipulate the thermo-solutal dynamics in smart electrically conducting functional liquids such as electroconductive polymers. Flow patterns are significantly influenced by the intensity and organization of the magnetic field in the fluid. The magnetic field exerts an effect on the colloidal 
fluid particles, altering the fluid's concentration and heat transmission characteristics. A magnetic nanofluid is a nanofluid with electrically conducting properties imparted by nanoparticles which permit the liquid to be manipulated by an external magnetic field. Flows of magnetic nanofluids can be simulated by combining magnetohydrodynamics of viscous fluids with nanofluid models such as the Buongiorno model. Hayat et al. [22] computed the influence of magnetic field on the flow of Casson magnetic nanofluid over a stretching surface. Bhatti et al. [23] evaluated the impact of thermodiffusion and diffusion thermal parameters on a hyperbolic tangent magnetized nanofluid under the effect of Hall current. Khan et al. [24] investigated computationally the nonlinear thermal radiation flux effects on hydromagnetic Sisko nanofluid flow with convective boundary conditions.

The above studies did not simultaneously consider microstructural and nanofluid characteristics in magnetohydrodynamic flow from a stretching surface in a porous medium. Motivated by developing a more comprehensive model for actual magnetic non-Newtonian nanofluids, the current work investigates the radiative-convective chemically reacting flow of magnetized nanofluid over a vertical stretching surface to a porous medium with thermal and solutal stratification, heat source, and viscous heating effects. The Rosseland flux model is used for thermal radiation, Eringen's micropolar fluid model is deployed for microstructural rheological features, and the Buongiorno nanoscale model is utilized to simulate nanoscale effects. The novelty of the present article is therefore the simultaneous consideration of multiple effectschemical reaction, thermal radiation, double stratification, micropolar non-Newtonian, and nanofluid characteristics in magnetohydrodynamic stretching sheet flow. To solve the novel boundary value problem, a semi-analytical/numerical approach is employed, i.e., Adomian decomposition method
(ADM), is used in collaboration with Padé approximants. Graphs and tables are used to illustrate the impact of key parameters on heat, mass, and momentum characteristics. Extensive interpretation of the results is included from a physical viewpoint. Comparison of the ADM-Padé approach with numerical Runge-Kutta quadrature is also included. The simulations are relevant to magnetic nano-polymer coating fabrication processes.

\section{Mathematical Model}

We consider two-dimensional steady incompressible MHD chemically reacting micropolar nanofluid boundary layer coating flow over a vertical stretching surface to a Darcian isotropic porous medium under thermal and species buoyancy effects in an $(x, y)$ coordinate system. Thermal and solutal stratifications, as well as nonlinear thermal radiation, are all taken into account. The $x$-axis is plotted along the vertical surface and the $y$-axis is normal to this (Figure 1). A uniform magnetic field of strength $B_{0}$ is applied transverse to the vertical surface, invoking a Lorentzian magnetic body force along the surface plane. The vertical sheet stretches with a linear velocity $U_{w}=a x$ where $a>0$ represents the stretching rate constant. Hall current and Ohmic dissipation are ignored. However, a heat source is present and viscous dissipation is included. A first-order homogenous chemical reaction (destructive) is considered. Buongiorno's model is used for the nanofluid and Eringen's micropolar model for microstructural rheology characteristics.

Under the above assumptions, the governing boundary layer conservation equations for mass, momentum, angular momentum (microrotation), energy, and nanoparticle species (conservation) for the regime may be shown to take the following form:

$$
\begin{aligned}
\frac{\partial u}{\partial x}+\frac{\partial v}{\partial y}= & 0 \\
\rho_{f}\left(u \frac{\partial u}{\partial x}+v \frac{\partial u}{\partial y}\right)= & (\mu+k) \frac{\partial^{2} u}{\partial y^{2}}+k \frac{\partial N}{\partial y}-\left(\sigma B_{0}^{2}+\frac{\mu}{k_{p}^{*}}\right) u \\
& +\rho_{f} \beta_{T} g\left(1-C_{\infty}\right)\left(T-T_{\infty}\right)+\beta_{c} g\left(\rho_{p}-\rho_{f}\right)\left(C-C_{\infty}\right), \\
\rho_{f} j\left(u \frac{\partial N}{\partial x}+v \frac{\partial N}{\partial y}\right)= & \gamma \frac{\partial^{2} N}{\partial y^{2}}-k\left(2 N+\frac{\partial u}{\partial y}\right), \\
u \frac{\partial T}{\partial x}+v \frac{\partial T}{\partial y}= & \alpha_{f} \frac{\partial^{2} T}{\partial y^{2}}+\tau\left[D_{B}\left(\frac{\partial C}{\partial y} \frac{\partial T}{\partial y}\right)+\frac{D_{T}}{T_{\infty}}\left(\frac{\partial T}{\partial y}\right)^{2}\right] \\
& -\frac{1}{\left(\rho c_{p}\right)_{f}} \frac{\partial q_{r}}{\partial y}+\frac{(\mu+k)}{\left(\rho c_{p}\right)_{f}}\left(\frac{\partial u}{\partial y}\right)^{2}+\frac{Q}{\left(\rho c_{p}\right)_{f}}\left(T-T_{\infty}\right), \\
u \frac{\partial C}{\partial x}+v \frac{\partial C}{\partial y}= & D_{B} \frac{\partial^{2} C}{\partial y^{2}}+\frac{D_{T}}{T_{\infty}} \frac{\partial^{2} T}{\partial y^{2}}-K_{r}^{*}\left(C-C_{\infty}\right) .
\end{aligned}
$$




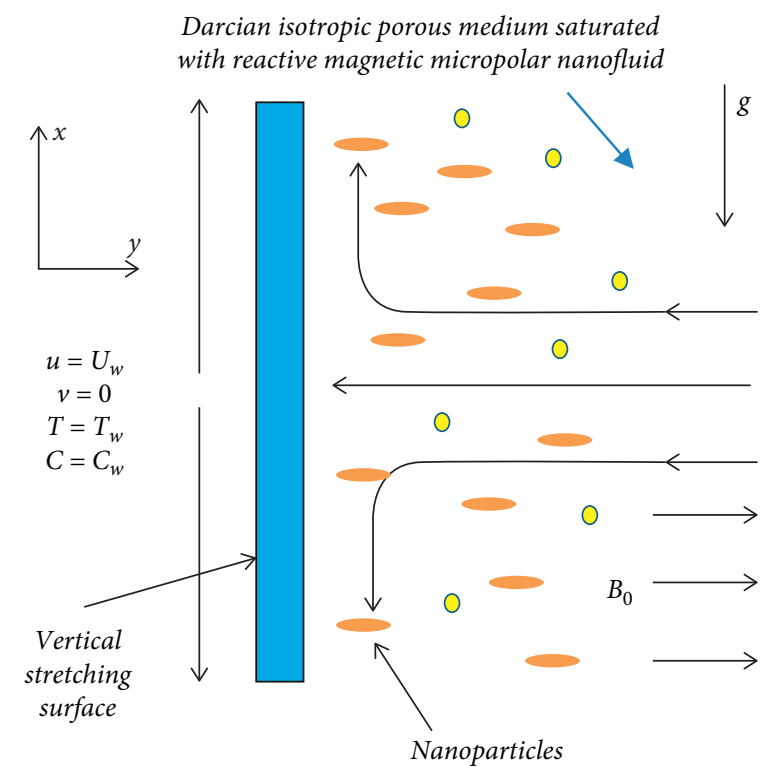

FIGURE 1: Geometrical configuration.

The relevant boundary conditions at the surface (wall) and in the free stream are prescribed as

$$
\left.\begin{array}{ll}
u=U_{w}=a x, v=0, N=-m \frac{\partial u}{\partial y}, T=T_{w}=T_{0}+b x, C=C_{w}=C_{0}+c x, & \text { at } y=0, \\
u \longrightarrow 0, N \longrightarrow 0, T \longrightarrow T_{\infty}=T_{0}+d x, C \longrightarrow C_{\infty}=C_{0}+e x, & \text { as } y \longrightarrow \infty
\end{array}\right\} .
$$

Here, equation (1) indicates the mass conservation equation i.e. the equation of continuity. Equation (2) is the linear momentum conservation equation. Equation (3) is the angular momentum conservation equation. Equations (4) and (5) are the energy and mass (solutal) conservations equations, respectively. The left-hand sides of each of equations (2)-(5) represent the convective derivative and the first term on the right-hand side terms denotes diffusion term for the corresponding profiles. In the Eringen micropolar model, there are two different viscosities, i.e., fluid dynamic viscosity and vortex viscosity. The third term of equation (2) is due to the resistivity offered by the magnetic field and the Darcian drag due to the porous medium fiber impedance. The last two terms represent the mixed convection effects dictated by both thermal and solutal buoyancy. Equation (3), i.e., the microrotation/angular momentum conservation, includes spin effects of the microelements. The third term on the right-hand side of the energy conservation equation (4) is the thermal radiation flux term
(Rosseland), the fourth term indicates the coupling effect of viscous dissipation, and the last term is the additional heat source/sink. Further, the $2^{\text {nd }}$ term on the right-hand side of the species (concentration) conservation equation (5) is due to thermal diffusion and the last term represents a first-order chemical reaction effect. Following Rosseland's approximation [25] for nonlinear thermal radiation, the net radiation heat flux can be defined as follows:

$$
\begin{gathered}
q_{r}=-\frac{4 \sigma^{*}}{3 k^{*}} \frac{\partial T^{4}}{\partial y}=-\frac{16 \sigma^{*}}{3 k^{*}} \frac{T^{3} \partial T}{\partial y}, \\
\frac{\partial q_{r}}{\partial y}=-\frac{16 \sigma^{*}}{3 k^{*}} T^{3} \frac{\partial^{2} T}{\partial y^{2}}-\frac{16 \sigma^{*}}{k^{*}} T^{2}\left(\frac{\partial T}{\partial y}\right)^{2} .
\end{gathered}
$$

Implementing in the energy boundary layer equation (4) leads to

$$
\begin{aligned}
u \frac{\partial T}{\partial x}+v \frac{\partial T}{\partial y}= & \frac{\partial}{\partial y}\left[\left(\alpha_{f}+\frac{16 \sigma^{*}}{3 k^{*}} \frac{T^{3}}{\left(\rho c_{p}\right)_{f}}\right) \frac{\partial T}{\partial y}\right] \\
& +\tau\left[D_{B}\left(\frac{\partial C}{\partial y} \frac{\partial T}{\partial y}\right)+\frac{D_{T}}{T_{\infty}}\left(\frac{\partial T}{\partial y}\right)^{2}\right]+\frac{(\mu+k)}{\left(\rho c_{p}\right)_{f}}\left(\frac{\partial u}{\partial y}\right)^{2}+\frac{Q}{\left(\rho c_{p}\right)_{f}}\left(T-T_{\infty}\right)
\end{aligned}
$$


We introduce the following similarity transformations:

$$
\begin{aligned}
\eta & =\sqrt{\frac{a}{v_{f}}} y, \\
u & =a x f^{\prime}(\eta), \\
v & =-\sqrt{a v_{f}} f(\eta), \\
N & =a x \sqrt{\frac{a}{v_{f}}} \omega(\eta), \\
\theta(\eta) & =\frac{T-T_{\infty}}{T_{w}-T_{\infty}}, \\
\phi(\eta) & =\frac{C-C_{\infty}}{C_{w}-C_{\infty}} .
\end{aligned}
$$

Substitution of equations (10) in (2)-(9) generates the transformed dimensionless boundary layer equations for linear momentum, angular momentum, energy, and species, with associated boundary conditions, as follows:

$$
\begin{aligned}
& (1+K) f^{\prime \prime \prime}+f f^{\prime \prime}-f^{\prime 2}-\left(M+K p^{-1}\right) f^{\prime}+K \omega^{\prime}+\lambda(\theta+\delta \phi)=0 \\
& \left(1+\frac{K}{2}\right) \omega^{\prime \prime}+f \omega^{\prime}-f^{\prime} \omega-K\left(2 \omega+f^{\prime \prime}\right)=0, \\
& {\left[\left(1+R d(1+(\operatorname{Tr}-1) \theta)^{3}\right) \theta^{\prime}\right]^{\prime}+\operatorname{Pr}\left[f \theta^{\prime}+N b \theta^{\prime} \phi^{\prime}+N t \theta^{2 \prime}+S \theta\right]+(1+K) E c \operatorname{Pr} f^{\prime \prime 2}=0} \\
& \phi^{\prime \prime}+f \phi^{\prime}+\frac{N t}{N b} \theta^{\prime \prime}-\operatorname{Pr} L e K c \phi=0 \\
& \left.\begin{array}{ll}
f=0, f^{\prime}=1, \omega=-m f^{\prime \prime}, \theta=1-t, \phi=1-s, & \text { at } \eta=0, \\
f^{\prime} \longrightarrow 0, \omega \longrightarrow 0, \theta \longrightarrow 0, \phi \longrightarrow 0, & \text { as } \eta \longrightarrow \infty
\end{array}\right\} .
\end{aligned}
$$

In equations (11)-(14) the nondimensional parameters arising are

$$
\begin{aligned}
& K=\frac{k}{\mu}, M=\frac{\sigma B_{0}^{2}}{\rho_{f} a}, K p^{-1}=\frac{v_{f}}{a k_{p}^{*}}, \lambda=\frac{G r}{\operatorname{Re}^{2}}, G r=\frac{g \beta_{t}\left(1-C_{\infty}\right)\left(T_{w}-T_{\infty}\right) x^{3}}{v_{f}^{2}}, \operatorname{Re}=\frac{a x^{2}}{v_{f}}, \\
& \left.\begin{array}{l}
\delta=\frac{\beta_{c}\left(\rho_{p}-\rho_{f}\right)\left(C_{w}-C_{\infty}\right)}{\beta_{t}\left(1-C_{\infty}\right)\left(T_{w}-T_{\infty}\right) \rho_{f}}, R d=\frac{16 \sigma^{*} T_{\infty}^{3}}{3 k^{*} k_{f}}, \operatorname{Tr}=\frac{T_{w}}{T_{\infty}}, \operatorname{Pr}=\frac{v_{f}}{\alpha_{f}}, S=\frac{Q}{a\left(\rho c_{p}\right)_{f}}, t=\frac{d}{b}, \\
N b=\frac{\tau D_{B}\left(C_{w}-C_{\infty}\right)}{v_{f}}, N t=\frac{\tau D_{T}\left(T_{w}-T_{\infty}\right)}{T_{\infty} v_{f}}, E c=\frac{a^{2} x^{2}}{c_{p_{f}}\left(T_{w}-T_{\infty}\right)}, L e=\frac{\alpha_{f}}{D_{B}}, K c=\frac{K_{r}^{*}}{a}, s=\frac{e}{c}
\end{array}\right\} .
\end{aligned}
$$


All parameters are defined in the nomenclature.

\section{Physical Quantities of Engineering Interest}

The skin friction $\left(C_{f}\right)$, wall couple stress coefficient $\left(C_{s}\right)$, Nusselt number $\left(N u_{x}\right)$, and Sherwood number $\left(S h_{x}\right)$ are important quantities for materials processing operations. These take the following form:

$$
\begin{aligned}
C_{f} & =\frac{\tau_{w}}{(1 / 2) \rho_{f} U_{w}^{2}}, \\
C_{s} & =\frac{c_{w}}{\mu U_{w}}, \\
N u_{x} & =\frac{x q_{w}}{k_{f}\left(T_{w}-T_{\infty}\right)}, \\
S h_{x} & =\frac{x j_{m}}{D_{B}\left(C_{w}-C_{\infty}\right)} .
\end{aligned}
$$

Here,

$$
\begin{aligned}
\tau_{w} & =\left[(\mu+k) \frac{\partial u}{\partial y}+k N\right]_{y=0}, \\
c_{w} & =\gamma\left[\frac{\partial N}{\partial y}\right]_{y=0}, \\
j_{m} & =-D_{B}\left[\frac{\partial C}{\partial y}\right]_{y=0}, \\
q_{m} & =\left[-k_{f} \frac{\partial T}{\partial y}+q_{r}\right]_{y=0} \\
\gamma & =\mu\left(1+\frac{K}{2}\right) j, \\
j & =\frac{v}{a}, \\
\sqrt{\operatorname{Re}} C_{f} & =[1+(1-m) K] f^{\prime \prime}(0), \\
C_{s} & =\left(1+\frac{K}{2}\right) \omega^{\prime}(0), \\
\frac{N u_{x}}{\sqrt{\operatorname{Re}}} & =-\left[1+R d(1-(T r-1) \theta(0))^{3}\right] \theta^{\prime}(0), \\
\frac{S h_{x}}{\sqrt{\operatorname{Re}}} & =-\phi^{\prime}(0) .
\end{aligned}
$$

\section{Solution of the Nonlinear Boundary Value Problem}

4.1. Adomian Decomposition Method. Adomian [26] developed a robust semi-analytical method to solve linear and nonlinear differential/algebraic equations named the Adomian decomposition method (ADM). This method also uses the concept of series solutions. ADM has been shown to be generally excellent for solving highly strong nonlinear scientific problems [27]. The principal objective of ADM is to achieve a unified series solution of linear/nonlinear ordinary differential equations (ODEs) or partial differential equations (PDEs) in initial and boundary value problems (IVPs and BVPs). In this method, the nonlinear operator is decomposed into a series of functions. The nonlinear coupled transformed ordinary differential equations (11)-(14) are solved semi-analytically using ADM. The details of the procedure of ADM for the present magnetic micropolar nanofluid stretching sheet problem are as follows:

To rewrite equations (11)-(14), we introduced the operators $L_{1}=\left(d^{3} / d \eta^{3}\right)(\bullet)$ and $L_{2}=d^{2} / d \eta^{2}(\bullet)$ and the inverse operators $(\bullet)=\int_{0}^{\eta} \int_{0}^{\eta}(\bullet) \mathrm{d} \eta \mathrm{d} \eta$. $L_{1}^{-1}(\bullet)=\int_{0}^{\eta} \int_{0}^{\eta} \int_{0}^{\eta}(\bullet) \mathrm{d} \eta \mathrm{d} \eta \mathrm{d} \eta L_{2}^{-1}$ Therefore, equations (11)-(14) can be expressed as

$$
\begin{gathered}
(1+K) L_{1}(f)=-f f^{\prime \prime}+f^{\prime 2}+\left(M+K p^{-1}\right) f^{\prime}-K \omega^{\prime}-\lambda(\theta+\delta \phi), \\
\left(1+\frac{K}{2}\right) L_{2}(\omega)=f^{\prime} \omega-f \omega^{\prime}+K\left(2 \omega+f^{\prime \prime}\right), \\
L_{2}(\theta)=-\left[\frac{3 R d(\operatorname{Tr}-1)\{1+(\operatorname{Tr}-1) \theta\}^{2} \theta^{\prime 2}+\operatorname{Pr}\left[f \theta^{\prime}+N b \theta^{\prime} \phi^{\prime}+N t \theta^{\prime 2}\right]+(1+K) E c \operatorname{Pr} f^{2 \prime \prime}+\operatorname{Pr} S \theta}{1+R d\{1+(\operatorname{Tr}-1) \theta\}^{3}}\right],
\end{gathered}
$$




$$
L_{2}(\phi)=\operatorname{Pr} L e K c \phi-f \phi^{\prime}-\frac{N t}{N b} \theta^{\prime \prime}
$$

Hence, taking the inverse of equations (20)-(23) we get

$f(\eta)=\frac{1}{1+K} L_{1}^{-1}\left\{f^{\prime 2}-f f^{\prime \prime}+\left(M+K p^{-1}\right) f^{\prime}-K \omega^{\prime}-\lambda(\theta+\delta \phi)\right\}$,

$\omega(\eta)=\frac{1}{(1+(K / 2))} L_{2}^{-1}\left\{f^{\prime} \omega-f \omega^{\prime}+K\left(2 \omega+f^{\prime \prime}\right)\right\}$,

$\theta(\eta)=-L_{2}^{-1}\left\{\frac{3 R d(\operatorname{Tr}-1)\{1+(\operatorname{Tr}-1) \theta\}^{2} \theta^{2 \prime}+\operatorname{Pr}\left[f \theta^{\prime}+N b \theta^{\prime} \phi^{\prime}+N t \theta^{2 \prime}\right]+(1+K) E c \operatorname{Pr} f^{2 \prime \prime}+\operatorname{Pr} S \theta}{1+R d\{1+(T r-1) \theta\}^{3}}\right\}$

$\phi(\eta)=L_{2}^{-1}\left\{\operatorname{Pr} L e K c \phi-f \phi^{\prime}-\frac{N t}{N b} \theta^{\prime \prime}\right\}$

The corresponding initial conditions are

$$
\begin{aligned}
f(0) & =0, \\
f^{\prime}(0) & =1, \\
f^{\prime \prime}(0) & =p_{1}, \\
\omega(0) & =-m p_{1}, \\
\omega^{\prime}(0) & =p_{2}, \\
\theta(0) & =1-t, \\
\theta^{\prime}(0) & =p_{3}, \\
\phi(0) & =1-s, \\
\phi^{\prime}(0) & =p_{4} .
\end{aligned}
$$

The initial guess solutions as well as recursive expressions are as follows:

$$
\begin{aligned}
f_{0}(\eta) & =\eta+\frac{\eta^{2}}{2} p_{1}, \\
\omega_{0}(\eta) & =-m p_{1}+\eta p_{2}, \\
\theta_{0}(\eta) & =(1-t)+\eta p_{3}, \\
\phi_{0}(\eta) & =(1-s)+\eta p_{4}, \\
f_{m+1}(\eta) & =\frac{1}{1+K} L_{1}^{-1}\left\{f^{2,}-f f^{\prime \prime}+\left(M+K p^{-1}\right) f^{\prime}-K \omega^{\prime}-\lambda(\theta+\delta \phi)\right\}, \\
\omega_{m+1}(\eta) & =\frac{1}{(1+(K / 2))} L_{2}^{-1}\left\{f^{\prime} \omega-f \omega^{\prime}+K\left(2 \omega+f^{\prime \prime}\right)\right\}
\end{aligned}
$$




$$
\theta_{m+1}(\eta)=-L_{2}^{-1}\left\{\frac{3 R d(\operatorname{Tr}-1)\{1+(\operatorname{Tr}-1) \theta\}^{2} \theta^{2 \prime}+\operatorname{Pr}\left[f \theta^{\prime}+N b \theta^{\prime} \phi^{\prime}+N t \theta^{2 \prime}\right]+(1+K) E c \operatorname{Pr} f^{2 \prime \prime}+\operatorname{Pr} S \theta}{1+R d\{1+(\operatorname{Tr}-1) \theta\}^{3}}\right\},
$$

$$
\phi_{m+1}(\eta)=L_{2}^{-1}\left\{\operatorname{PrLeKc} \phi-f \phi^{\prime}-\frac{N t}{N b} \theta^{\prime \prime}\right\} .
$$

Equations (35) and (36) can be simplified by truncating the binomial expansion of the denominator of equation (35). Assuming all the linear as well as nonlinear terms of the right-hand side of equations (33)-(36) in series form and taking $m=0,1,2, \ldots$, the recursive relations (33)-(36) can be solved. Evaluating the unknowns numerically using the MATLAB BVP solver and taking some specific values of pertinent parameters as $K=0.1, M=1, K p=0.2$, $\lambda=5, \delta=3, \quad \operatorname{Tr}=2, \operatorname{Pr}=2, S=0.01, E c=0.5, N b=0.5$, $N t=0.5, L e=3, \quad t=0.5, R d=2, K c=1, m=0.5, \quad$ and $s=0.5$, we get the approximate analytical solutions for stream function, microrotation, temperature, and nanoparticle concentration profiles as follows:

$$
\begin{aligned}
f(\eta)= & \left\{\eta-1.07505 \eta^{2}+1.944 \eta^{3}-1.24795 \eta^{4}+1.11907 \eta^{5}-1.01231 \eta^{6}+0.87583 \eta^{7}-0.4367 \eta^{8}\right. \\
+ & 0.08453 \eta^{9}-0.00598 \eta^{10}+0.0001 \eta^{11}, \\
\omega(\eta)= & 1.07505+1.0774 \eta+0.53752 \eta^{2}+1.20007 \eta^{3}-1.06184 \eta^{4}+0.00261 \eta^{5}+0.04127 \eta^{6} \\
+ & 0.03194 \eta^{7}+0.01546 \eta^{8}-0.001593 \eta^{9}-0.00008 \eta^{10}, \\
\theta(\eta)= & 0.5+1.86154 \eta-3.84474 \eta^{2}-1.56 \eta^{3}-11.63709 \eta^{4}+24.37027 \eta^{5}+80.5245 \eta^{6} \\
- & 189.281 \eta^{7}+313.405 \eta^{8}-498.357 \eta^{9}-47.85 \eta^{10}+12.456 \eta^{11}-1479.3385 \eta^{12} \\
& +377.8 \eta^{13}-565.5 \eta^{14}-3209.4 \eta^{15}+130.6 \eta^{16}-1309.12 \eta^{17}-3571.4 \eta^{18}-1261.499 \eta^{19}, \\
\phi(\eta)= & 0.5-1.557 \eta+2.423 \eta^{2}+0.5204 \eta^{3}+6.0827 \eta^{4}-12.133 \eta^{5}-40.6603 \eta^{6} \\
& +94.785 \eta^{7}-156.44 \eta^{8}+248.9 \eta^{9}+23.82 \eta^{10}-6.1 \eta^{11} 739.6 \eta^{12}-188.9 \eta^{13} \\
& +282.8 \eta^{14}+1604.7 \eta^{15}+65.3 \eta^{16}+654.56 \eta^{17}+1785.7 \eta^{18}+630.8 \eta^{19}+1784.4 \eta^{20} .
\end{aligned}
$$

All the functions and coefficients are used in truncated forms. To increase the accuracy in the solution of the present boundary value problem, we have adopted Padé approximants after applying ADM.

4.2. Padé Approximant. A Padé approximation of $f(\eta)$ on $[a, b]$ can be expressed as a rational function that contains two polynomials, say $P_{N}(\eta)$ as the numerator and $Q_{M}(\eta)$ a denominator of degrees $N$ and $M$, respectively [28]. The notation $[N / M]$ is used to denote the quotient. To obtain better results, we have combined the differential transform method and the diagonal Padé approximants $[N / N]$ and used them in the process. Padé approximants are widely used (Baker and Morris, [28]) to accelerate the convergence of a given series. Arain et al. [29] also used Padé approximants in conjunction with a differential transform method (DTM) to compute the viscoelastic magnetic nanofluid flow between rotating disks. We have considered the function $f(\eta)$ in power series form as

$$
f(\eta)=\sum_{i=0}^{\infty} c_{i} \eta^{i}
$$

The notation $c_{i}, i=0,1,2, \ldots$, represents the coefficients of the power series\#, and $f(\eta)$ is the associated function. The $[L / M]$ Padé approximant is a rational fraction defined by

$$
f(\eta)=\frac{a_{0}+a_{1} \eta+a_{2} \eta^{2} \cdots \cdots+a_{L} \eta^{L}}{b_{0}+b_{1} \eta+b_{2} \eta^{2} \cdots \cdots+b_{M} \eta^{M}} .
$$

The Maclaurin series expansion of equation (42) agrees with equation (41). In equation (42), a number of coefficients in the numerator and denominator are $L+1$ and $M+1$, respectively. These numbers suggest $[L / M]$ is to fit the series expression in equation (41) with orders $1, \eta, \eta^{2}, \ldots, \eta^{L+M}$. In the notation of formal power series,

$$
\sum_{i=0}^{\infty} c_{i} \eta^{i}=\frac{a_{0}+a_{1} \eta+a_{2} \eta^{2}+\cdots+a_{L} \eta^{L}}{b_{0}+b_{1} \eta+b_{2} \eta^{2}+\cdots+b_{M} \eta^{M}}+0\left(\eta^{L+M}\right) .
$$

Equating the coefficients of $\eta^{L+1}, \eta^{L+2}, \ldots, \eta^{L+M}$, we get 


$$
\left.\begin{array}{l}
b_{M} c_{L-M+1}+b_{M-1} c_{L-M+2}+\cdots+b_{0} c_{L+1}=0 \\
b_{M} c_{L-M+2}+b_{M-1} c_{L-M+3}+\cdots+b_{0} c_{L+2}=0 \\
\cdots \\
\quad b_{M} c_{L}+b_{M-1} c_{L+1}+\cdots+b_{0} c_{L+M}=0
\end{array}\right\} .
$$

Taking $i<0, c_{i}=0$ and $b_{0}=1$, equation (44) can be written in matrix form as

$$
\left(\begin{array}{ccc}
c_{L-M+1} & c_{L-M+2 \ldots} \ldots & c_{L+1} \\
c_{L-M+2} & c_{L-M+3 \ldots} \ldots & c_{L+2} \\
c_{L} & c_{L+1 \ldots} & c_{L+3}
\end{array}\right)\left(\begin{array}{c}
b_{M} \\
b_{M-1} \\
\ldots \\
b_{0}
\end{array}\right)=-\left(\begin{array}{c}
c_{L+1} \\
c_{L+2} \\
\ldots \\
c_{L+M}
\end{array}\right) .
$$

The values of $b_{i}$ can be found from the above expression and from equation (43) we obtain

$$
\begin{aligned}
& a_{0}=c_{0}, \\
& a_{1}=c_{1}+b_{1} c_{0}, \\
& a_{2}=c_{2}+b_{1} c_{1}+b_{2} c_{0}, \ldots, \\
& a_{L}=c_{L}+\sum_{i=1}^{\min [L / M]} b_{i} c_{L-i} .
\end{aligned}
$$

Combining equations (43) and (45) enables the determination of the coefficients of the numerator and denominator which are called Padé equations. The diagonal Padé approximations $[L / L]$ are as follows:

$$
\begin{aligned}
& f_{P A D E}[2 / 2]=\frac{\eta-2.14283 \eta^{2}}{1-1.06783 \eta-3.09232 \eta^{2}}, \\
& f_{P A D E}[3 / 3]=\frac{\eta-0.611151 \eta^{2}+1.23251 \eta^{3}}{1+0.463849 \eta-0.213254 \eta^{2}+0.116795 \eta^{3}}, \\
& \omega_{P A D E}[2 / 2]=\frac{1.075-0.744369 \eta-1.57866 \eta^{2}}{1+0.309425 \eta+1.27806 \eta^{2}}, \\
& \omega_{P A D E}[3 / 3]=\frac{1.075+0.0814601 \eta+0.1195197 \eta^{2}+1.658441 \eta^{3}}{1+1.07765 \eta+0.690438 \eta^{2}+0.569551 \eta^{3}}, \\
& \theta_{P A D E}[2 / 2]=\frac{0.5+1.07996 \eta-7.95329 \eta^{2}}{1-1.56407 \eta-2.39198 \eta^{2}}, \\
& \theta_{P A D E}[3 / 3]=\frac{0.5+1.10348 \eta-5.46546 \eta^{2}+13.7339 \eta^{3}}{1-1.51704 \eta-2.40853 \eta^{2}+9.95237 \eta^{3}}, \\
& \phi_{P A D E}[2 / 2]=\frac{0.5-2.36152 \eta+3.84434 \eta^{2}}{1-1.60704 \eta-2.16487 \eta^{2}}, \\
& \phi_{P A D E}[3 / 3]=\frac{0.5-2.30314 \eta+6.25394 \eta^{2}-3.7424 \eta^{3}}{1-1.49028 \eta+3.01817 \eta^{2}+8.09971 \eta^{3}} .
\end{aligned}
$$

\section{Result and Discussion}

The graphical results produced for variation of several emerging parameters will be discussed in this section. Earlier, Ramzan et al. [30] validated the present computer programs by including certain additional parameters, such as the porosity parameter in the momentum equation, Eckert number, and heat source parameter in the energy equation. The solution process is a strong advantage of the present ADM approach. We use approximate analytical ADM solutions to refine each profile and thereafter deploy the Padé approximant of various orders to refine each profile. Both results are, however, compared to the traditional numerical technique (Runge-Kutta shooting method). Figure 2 shows comparison plot for all the profiles with solution obtained by ADM-Padé, ADM, and numerical shooting, with some default assigned values of the physical parameters $K=0.1, M=1, K p=0.2, \lambda=5, \delta=3, T r=2$, $\operatorname{Pr}=2, S=0.01, E c=0.5, N b=0.5, N t=0.5, L e=3$, $t=0.5, R d=2, K c=1, m=0.5$, and $s=0.5$. This indicates a good correlation between the convergence procedures of the methodologies employed in this study.

The comparison of the stream function, velocity, microrotation, temperature, and concentration profiles is illustrated in Figures 3-7 for the numerical method, Adomian decomposition method (ADM), and the novel semi- 


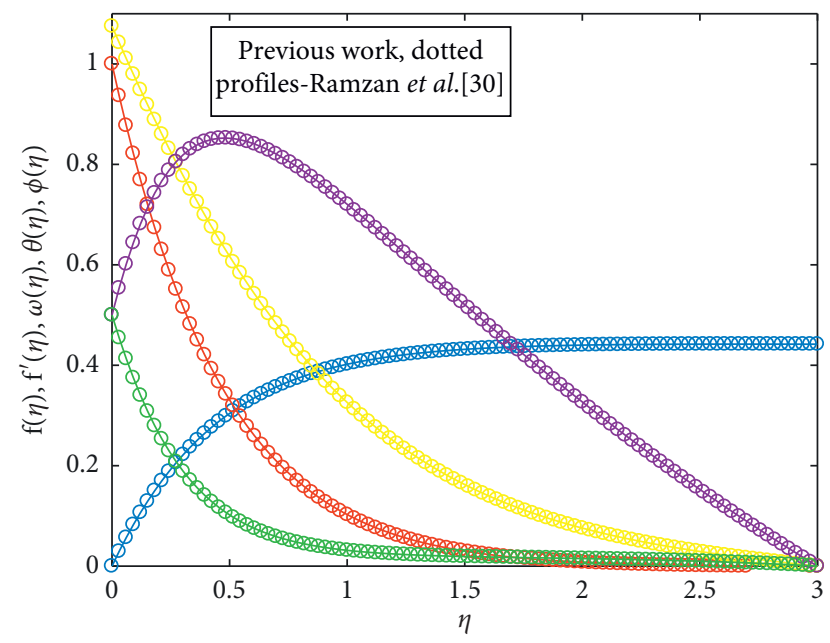

Previous work present

$$
\begin{array}{ll}
\odot \mathrm{f}(\eta) & -\theta(\eta) \\
-\mathrm{f}^{\prime}(\eta) & -\phi(\eta) \\
-\omega(\eta) &
\end{array}
$$

FiguRE 2: Validation of stream function, velocity, microrotation, temperature, and concentration profiles.

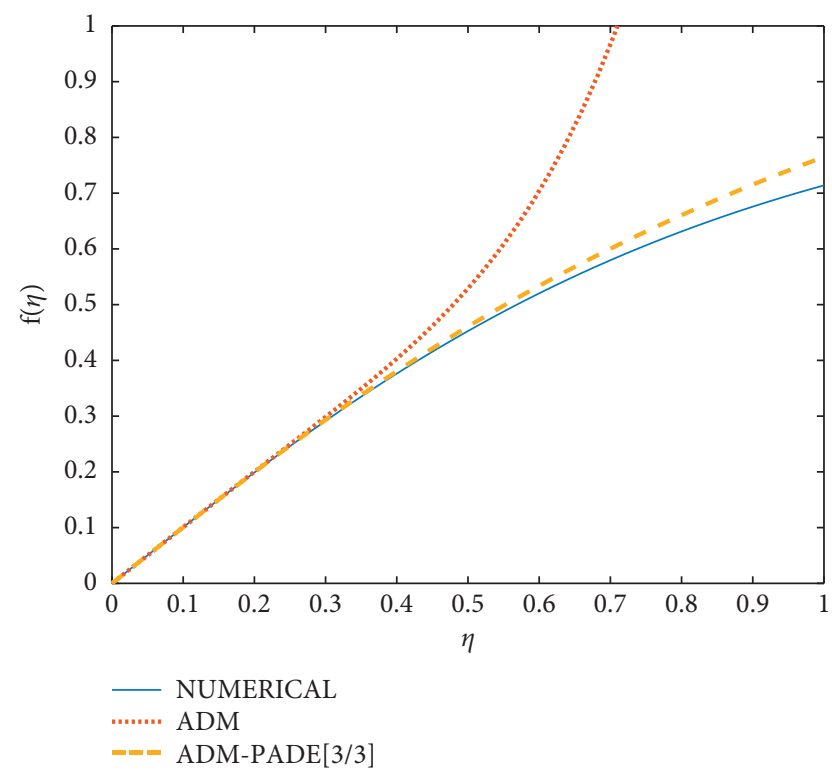

FIgURE 3: Comparative plot of stream function.

analytical method, ADM-Padé-approximant. These graphs show the importance of the Padé-Approximant method as it converges more rapidly than ADM on its own when compared with numerical methods such as the $4^{\text {th }}$-order Runge-Kutta method (shooting technique). This encouraged us to study the behavior of different profiles of a micropolar-nanofluid flow by deploying Padé-approximant after using ADM.

The study shows since $\mathrm{ADM}$ requires some computer programs to calculate the values of $f^{\prime \prime}(0), \omega^{\prime}(0), \theta^{\prime}(0)$, and $\phi^{\prime}(0)$ to find the initial solution, recurrence functions using

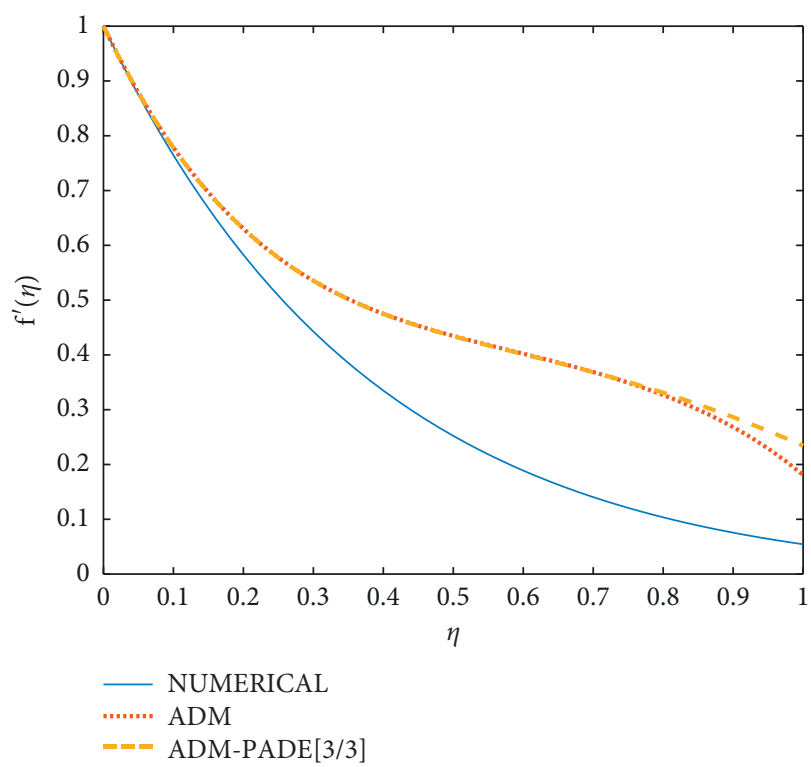

Figure 4: Comparative plot of velocity profile.

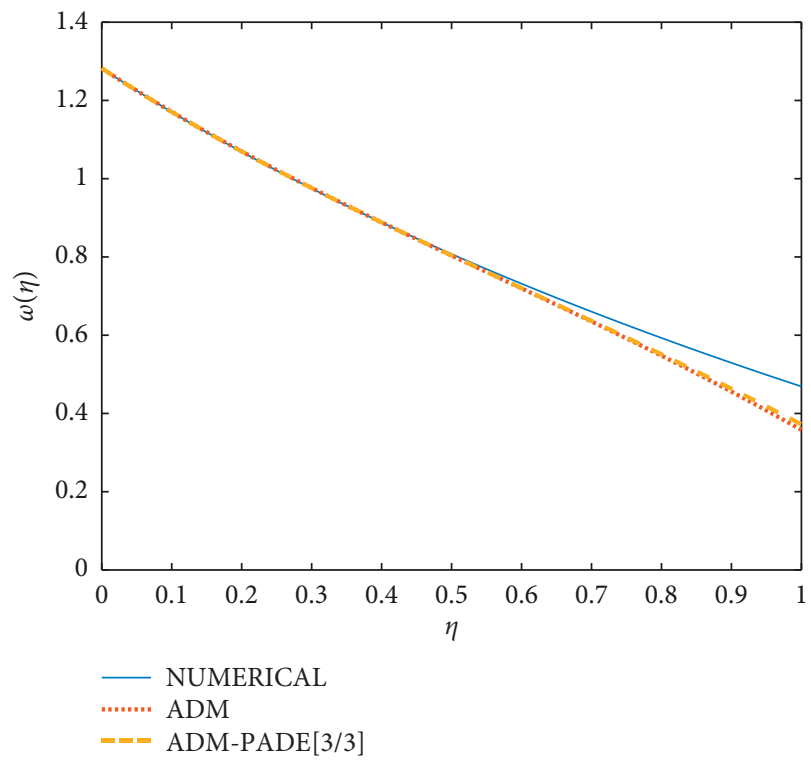

Figure 5: Comparative plot of microrotation profile.

these initial solutions give the final solutions as equations (37)-(40). After getting the recurrence solutions, the diagonal Padé Approximant of an order [3/3] is used for the graphical and tabular presentations. The study shows the diagonal Padé approximant gives accelerated convergent solutions. Figures 3-7 confirm these observations.

In Figure 8, the impacts of magnetic parameter $(M)$, microgyration parameter $(m)$, mixed convection parameter $(\lambda)$, and temperature difference parameter $(\delta)$ on velocity parameter have been studied. Figure 8 (a) shows that velocity is strongly suppressed with increasing magnetic parameter, $M$. Lorentz force $-(M) f^{\prime}$ is created by the imposition of the magnetic field on the conducting fluid, which retards the 


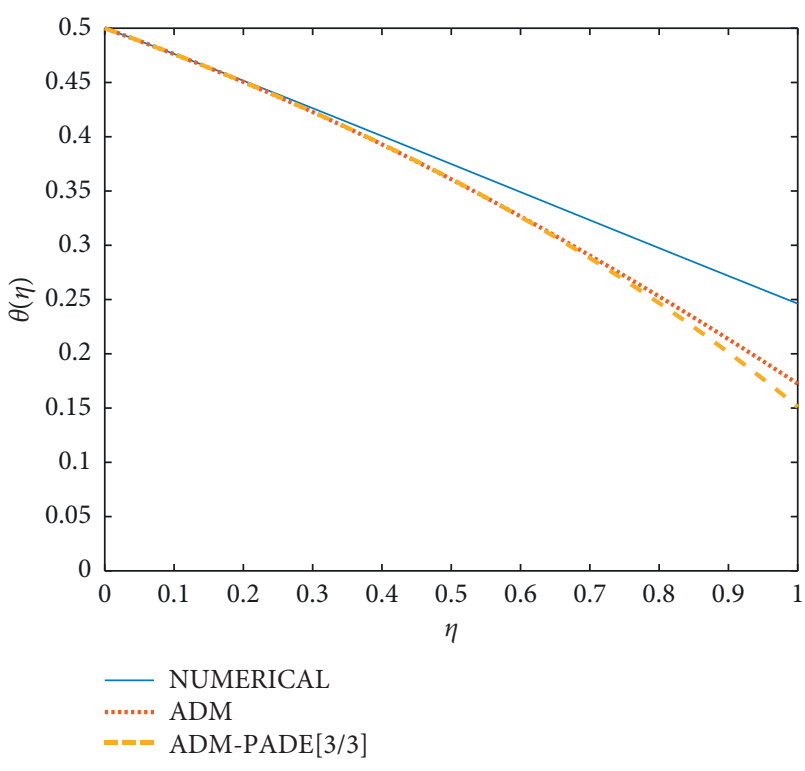

Figure 6: Comparative plot of temperature profile.

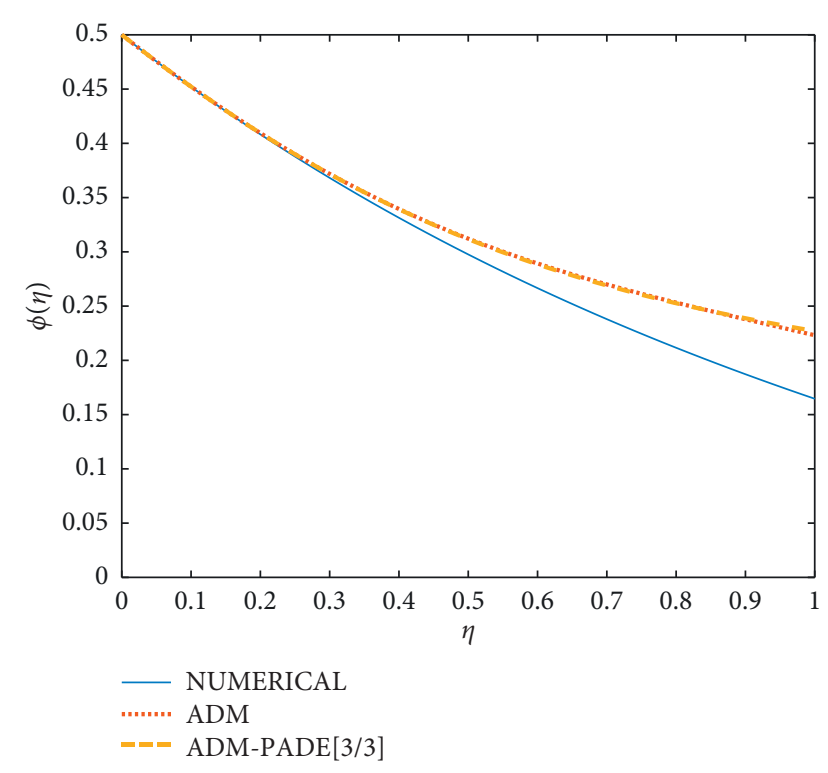

Figure 7: Comparative plot of concentration profile.

boundary layer flow and increases momentum boundary layer thickness. Higher values of $M$ clearly elevate Lorentz force which produces strong deceleration. The converse response is observed with large permeability parameter, $K p$. The Darcian body force is inversely proportional to $K p$ as observed in Figure 9(a), via the term. $-\left(M+K p^{-1}\right) f^{\prime}$ in the momentum equation (11). Higher $K p$ values imply greater permeability and therefore a decrease in solid matrix fibers in the porous medium. This reduces the Darcian drag force and accelerates the boundary layer flow leading to a decrease in momentum boundary layer thickness. Figure 8(b) shows that velocity is strongly damped with an increment in micropolar parameter, $m$. This parameter appears in the wall boundary condition (14) and relates to the nature of the microelements spin effect at the boundary. For the Newtonian case $m=0$, velocity is a maximum. Figure $8(\mathrm{c})$ shows that for increasing values of mixed convection parameter $(\lambda)$ that enhances the fluid velocity near the boundary for $\eta \leq 1$ and afterward it decelerates the fluid. Thermal buoyancy as simulated in the parameter $\lambda=\left(G r / R e^{2}\right)$ therefore has a complex relationship with the linear velocity field. Figure $8(\mathrm{~d})$ presents the influence of the buoyancy ratio parameter, i.e., $\delta=\left(\beta_{c}\left(\rho_{p}-\rho_{f}\right)\left(C_{w}-C_{\infty}\right) / \beta_{t}\left(1-C_{\infty}\right)\right.$ $\left.\left(T_{w}-T_{\infty}\right) \rho_{f}\right)$ on velocity evolution. This parameter expresses the relative contribution of solutal (nanoparticle species) buoyancy force to thermal buoyancy force. For negative values of $\delta$, these buoyancy forces oppose each other and for positive values they assist each other. It is apparent that for $\delta=-3$ there is a strong acceleration in the flow (linear profiles) whereas the opposite trend is computed for $\delta=+3$ (parabolic profiles). These trends are sustained at all values of transverse coordinate, $\delta$.

Figures 9(a)-9(c) visualize the velocity evolution with different values of the micropolar material parameter $(K)$, Prandtl number $(\mathrm{Pr})$, and thermal stratification parameter $(t)$. Here, non-zero values of material parameter $K$ indicate the non-Newtonian characteristics of the fluid. $K=(k / \mu)$ expresses the ratio of micropolar vortex viscosity to Newtonian dynamic viscosity. Momentum boundary layer thickness is clearly reduced with greater micropolar parameter since the velocity is elevated strongly at all values of transverse coordinate. With elevation in Prandtl number, there is a weak enhancement in velocity. At Prandtl number of 1 , both momentum and thermal diffusion rates are equal for the magnetic micropolar nanofluid. However, as $\operatorname{Pr}$ is increased, the thermal diffusion rate (and thermal conductivity) is decreased, and the momentum diffusion rate is much higher. This results in acceleration of the boundary layer flow and decrease in momentum boundary layer thickness. Higher values of Prandtl number $(\mathrm{Pr}=10)$ correspond to actual magnetic coating materials [18]. Figure 9(c) shows that as a thermal stratification parameter $(t=(d / b))$ increases, the sheet temperature reduces which induces a boost in the velocity profiles. This parameter features in the wall boundary condition, $\theta=1-t$, in equation (15) and clearly exerts a strong influence on velocity distribution. Greater thermal stratification produces a decrease in momentum boundary layer thickness in the stretching flow regime.

Figure 10 visualizes the impact of (a) $M$ (b) $m$ (c) $\lambda$ (d) $\delta$ on microrotation profile, i.e., angular velocity, in the presence/absence of $K p$. Maximum angular velocity is generally observed at the wall (sheet). With increment in magnetic parameter, $M$, there is a strong increase in microrotation, i.e., microelements spin faster in the magnetic micropolar nanofluid. This is maintained at all values of transverse coordinate. Greater permeability $K p$ however generates a reduction in angular velocity, i.e., suppresses the gyratory motion of microelements. Figure 10(b) also demonstrates that the microrotation is elevated significantly with an increase in micropolar surface parameter $(m)$. It is noteworthy that when the value of the microgyration parameter $(m)$ 


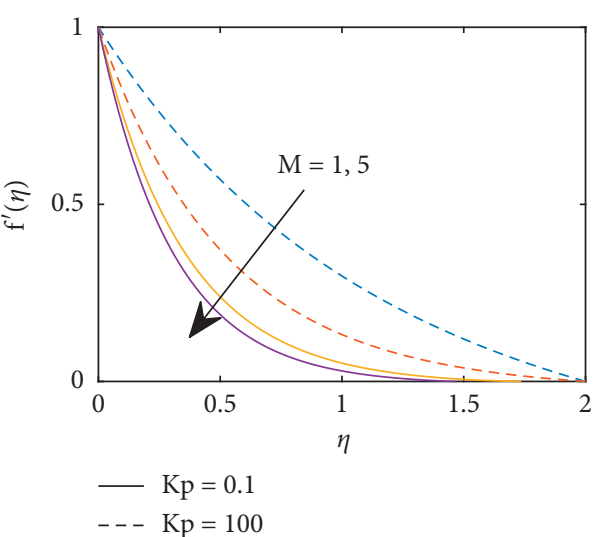

(a)

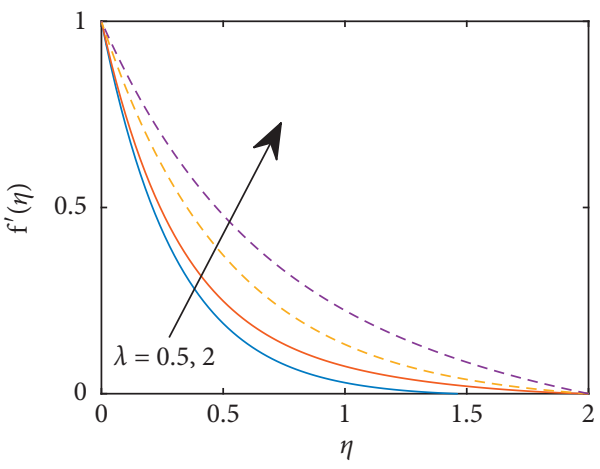

$\begin{aligned}-\mathrm{Kp} & =0.1 \\ ---\mathrm{Kp} & =100\end{aligned}$

(c)

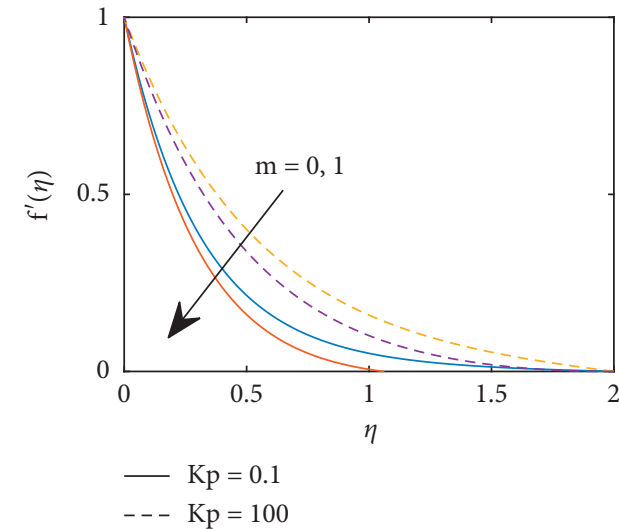

(b)

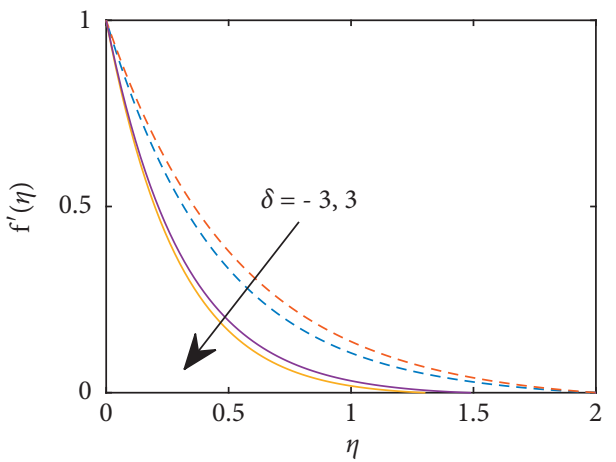

$-\mathrm{Kp}=0.1$

- - $\mathrm{Kp}=100$

FIgURE 8: Impact of (a) $M$, (b) $m$, (c) $\lambda$, (d) $\delta$ on velocity profile.

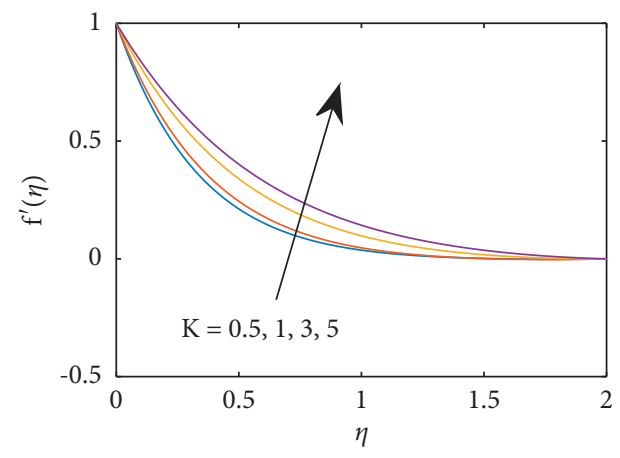

(a)

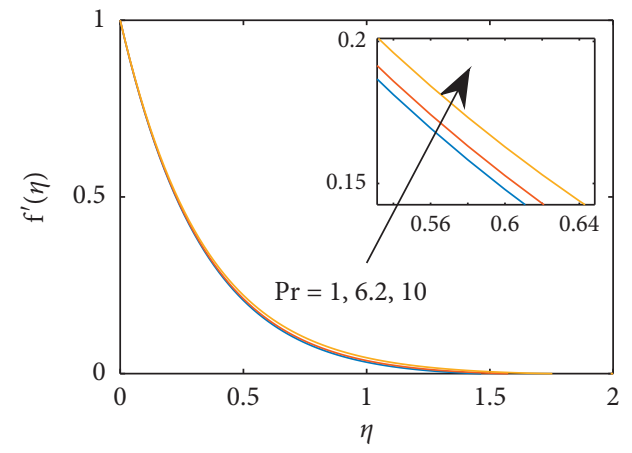

(b)

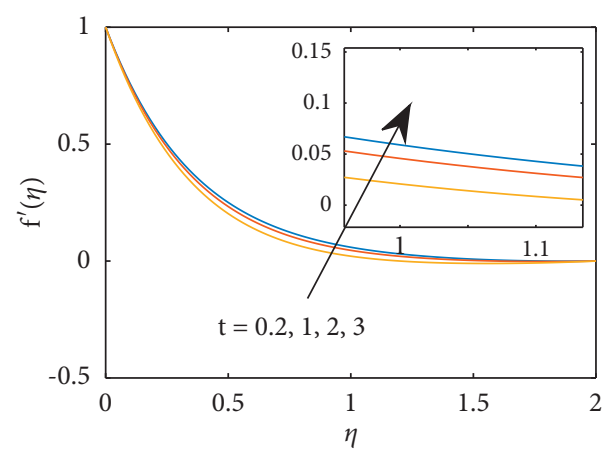

(c)

FIgURE 9: Impact of (a) $K$, (b) $\operatorname{Pr}$, (c) $t$ on velocity profile. 


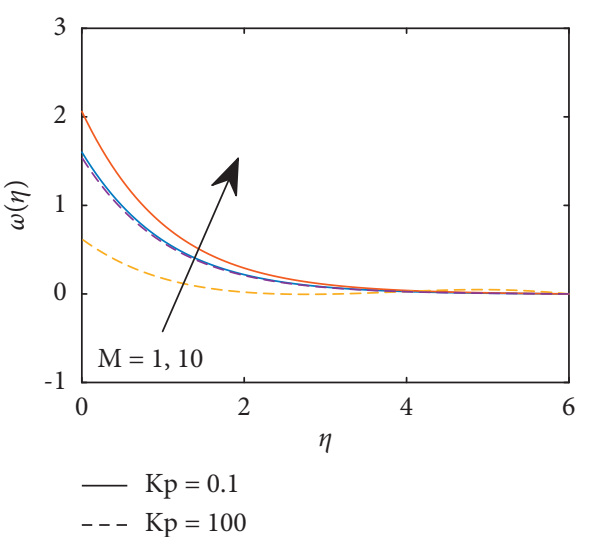

(a)

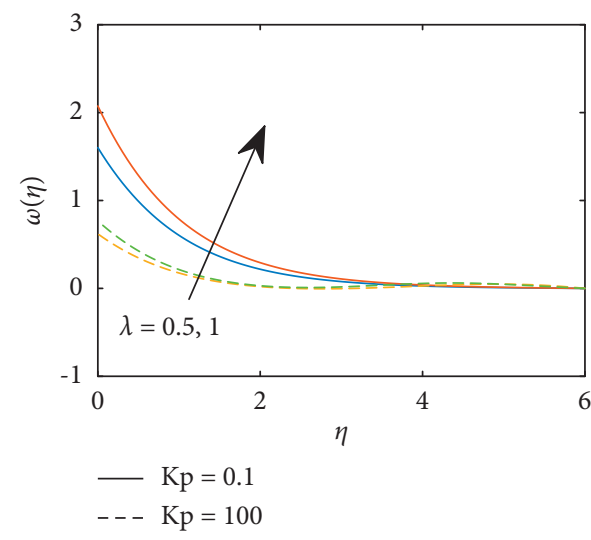

(c)

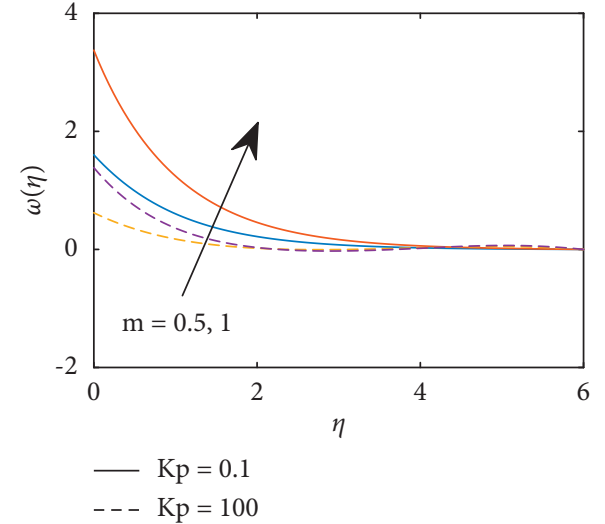

(b)

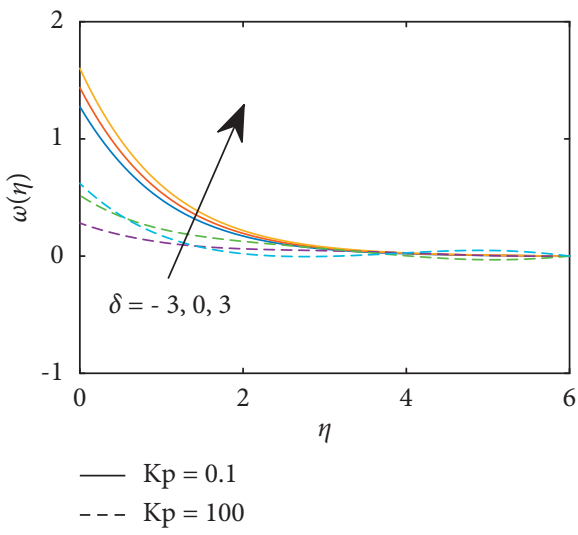

(d)

Figure 10: Impact of (a) $M$, (b) $m$, (c) $\lambda$, (d) $\delta$ on microrotation profile in presence $(K p=100) /$ absence $(K p=0.1)$ of $K p$.

increases from $m=0$, i.e., when microelements are unable to rotate (strong concentration) to $m=1$ (turbulent boundary layer flows), the microrotation profile is enhanced. Figure $10(\mathrm{c})$ shows that angular velocity is an increasing function of mixed convection parameter $(\lambda)$ in conjunction with the permeability parameter, Kp. Microrotation is therefore clearly elevated with greater mixed convection parameter values. Figure 10(d) shows that positive increment in buoyancy ratio parameter $(\delta)$ also produces an acceleration in the flow; i.e., the velocity is enhanced for the assisting thermal and species buoyancy case $(\delta>0)$ whereas it is reduced for the opposing buoyancy case $(\delta<0)$. It is also noteworthy that all profiles converge asymptotically to the free stream, confirming the imposition for an adequate infinity boundary condition in the ADM-Padé computations.

Figures 11(a)-11(c) illustrate the impact of micropolar vortex viscosity material parameter $(K)$, Prandtl number $(\operatorname{Pr})$, and thermal stratification parameter $(t)$ on angular velocity. Increasing values of $K$ are observed in Figure 11(a) to strongly damp the angular velocity profile, indicating that with increased vortex viscosity the microelements are constrained in their gyratory motion. The opposite effect is computed with increasing Prandtl number, for which in Figure 11(b), there is a boost in the angular velocity (microrotation). The greater momentum diffusivity relative to thermal diffusivity associated with higher Prandtl numbers assists the spin of microelements with reverse trend as expected in case of increasing higher values of Prandtl number (Pr). Figure 11(c) shows that the microrotation is depleted with greater thermal stratification parameter $(t=(d / b))$. As with other plots, the effect is most profound near the sheet surface (wall). The decrease in wall sheet temperature associated with higher thermal stratification reduces linear momentum and angular momentum in the boundary layer regime. Angular velocity is therefore supressed as microelement rotation is subdued.

Figure 12 portrays the impact of (a) thermal radiation, $R d$ (b) temperature ratio parameter, $T r$, (c) Prandtl number, and (d) heat source, $S$ on the temperature distribution. Figure 12(a) shows that the radiation from electromagnetic waves energizes the flow and elevates temperatures further from the sheet towards the free stream. However closer to the wall there is a decrement in temperatures with greater thermal radiation effect. With larger temperature ratio, as seen in Figure 12(b), temperature is suppressed near the wall whereas it is elevated significantly further from the wall. Increasing Prandtl number results in a decrease in temperatures computed further from the wall (Figure 12(c)). Increasing heat source parameter, $S$, clearly consistently boosts the temperatures at all locations from the wall to the free stream. This parameter represents hot spots in materials 


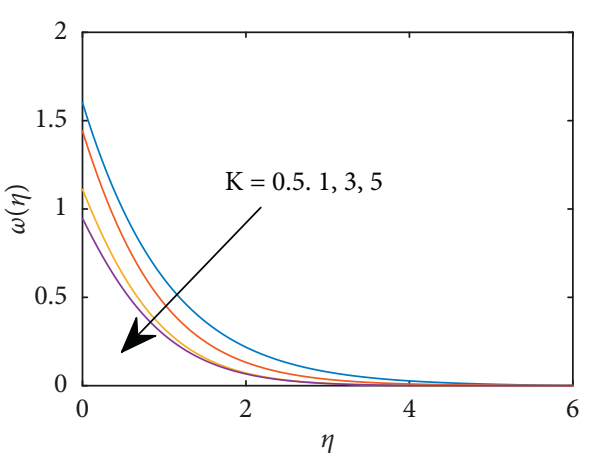

(a)

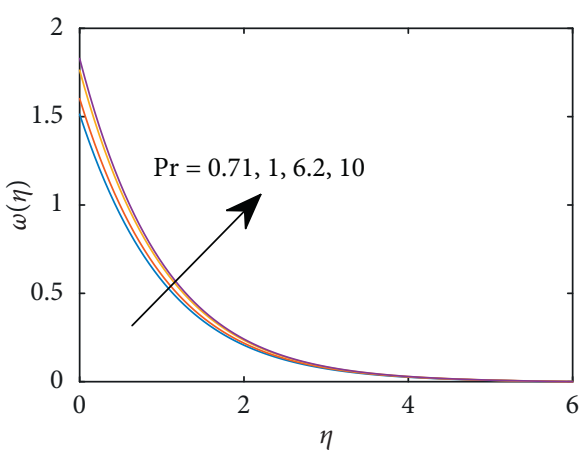

(b)

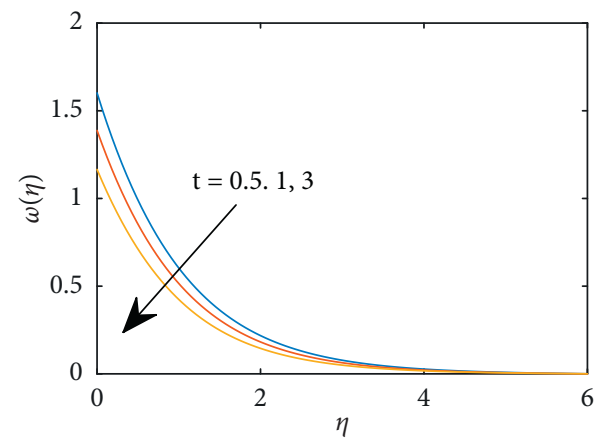

(c)

Figure 11: Impact of (a) $K$, (b) $\operatorname{Pr}$, (c) $t$ on microrotation profile.

processing which can be used to provide a boost in thermal energy during fabrication operations [18]. Thermal boundary layer thickness is elevated with greater heat source parameter. In all profiles, a temperature overshoot is also computed near the wall.

Figure 13 illustrates the influence of (a) $K$ and $t$ (b) $E c$ (c) $\mathrm{Nb}$ (d) $\mathrm{Nt}$ on temperature profiles. Figure 13(a) shows that with increment in stratification parameter $(t)$ temperatures are reduced and thermal boundary layer thickness is decreased. However, with increase in micropolar vortex parameter, $K$, temperatures are strongly enhanced, and thermal boundary layer thickness is increased. Increasing Eckert number, $E c$, in Figure 13(b) produces a strong elevation in temperatures. The viscous dissipation is boosted owing to a greater conversion of kinetic energy in the flow to frictional heat with greater Eckert number. This energizes the flow and increases thermal boundary layer thickness. The temperature peak is also progressively displaced further from the wall (sheet) with greater Eckert number. Figure 13(c) shows that, with greater Brownian motion parameter $(\mathrm{Nb})$, temperatures are initially suppressed near the wall whereas they are strongly elevated shortly thereafter, and this temperature enhancement is sustained towards the free stream. Thermal boundary layer thickness is therefore enhanced generally with greater random motion of the nanoparticles (Brownian dynamics). Figure 13(d) shows that with increasing thermophoresis parameter $(\mathrm{Nt})$ there is a sustained elevation in temperature at all locations from the wall to the free stream. The migration of nanoparticles under a temperature gradient is known as thermophoresis. This energizes the flow and increases thermal boundary layer thickness.

Figure 14 illustrates the influence of (a) Lewis number, $L e$ and thermal stratification parameter $t$ (b) chemical reaction parameter, $K c$ (c) Brownian motion parameter $N b$, and thermophoresis parameter $\mathrm{Nt}$ on nanoparticle concentration profiles. Figure 14(a) shows that there is a strong decrement in concentration magnitudes with greater Lewis number and also stratification parameter. The Lewis number is the ratio of thermal diffusivity with respect to mass diffusivity. With the increase in Lewis number, a slower diffusion process of the solutal concentration is encountered, resulting in the concentration boundary layer decreasing and a suppression in species (nanoparticle) diffusion rate. Figure 14(b) indicates that with an increase in chemical reaction parameters again there is a depletion in nanoparticle concentration values. The reaction is first-order homogenous and destructive, as simulated in the equation (14), via the term $-\operatorname{Pr} L e K c \phi$. This implies that nanoparticles react and are converted to another species. Therefore, physically the concentration of original nanoparticles, i.e., $\phi(\eta)$, must decrease with more intense chemical reaction effect (greater $K c$ values) as verified in Figure 14(b). Finally, Figure 14(c) visualizes the collective influence of $\mathrm{Nb}$ and $\mathrm{Nt}$ on nanoparticle concentrations. A boost is observed with greater thermophoresis effect whereas a decrement is computed with greater Brownian motion. The increased ballistic collisions of nanoparticles with higher $\mathrm{Nb}$ result in opposition to species diffusion which decreases concentrations and a thinner concentration boundary layer thickness 


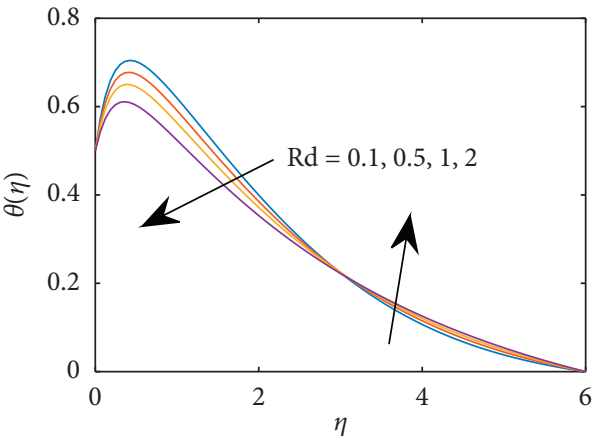

(a)

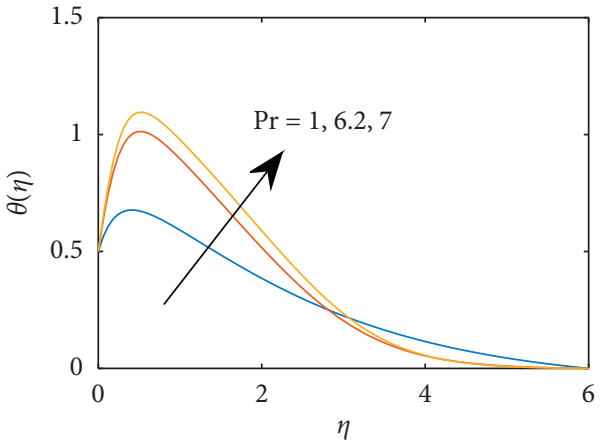

(c)

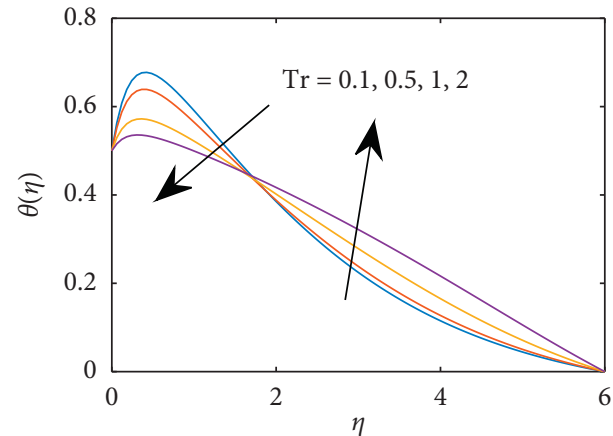

(b)

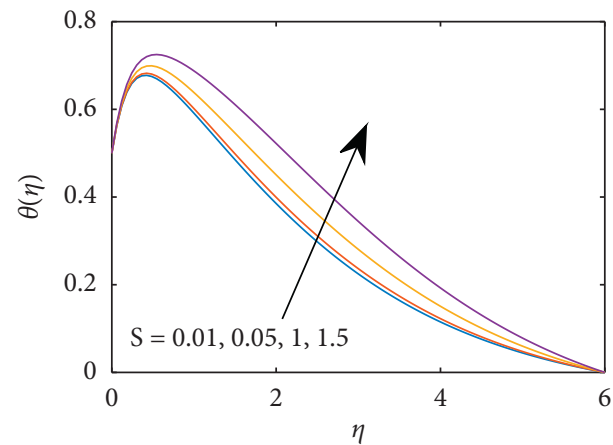

(d)

Figure 12: Impact of (a) $R d$, (b) $T r$, (c) $\operatorname{Pr}$, (d) $S$ on temperature profile.

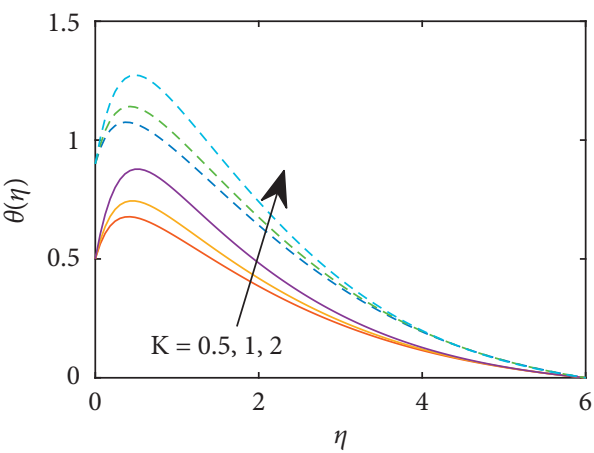

$-\mathrm{t}=0.5$

$---\mathrm{t}=0.1$

(a)

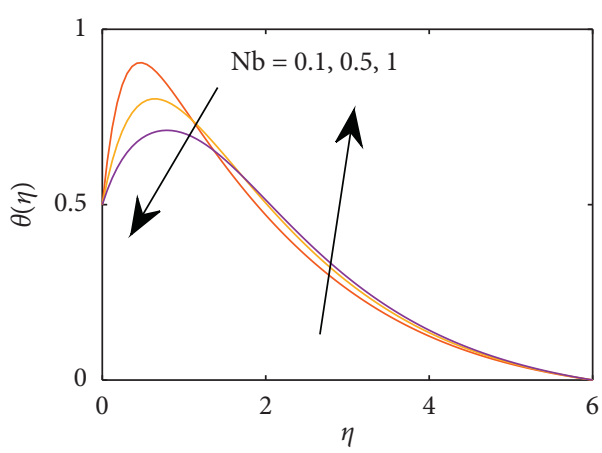

(c)

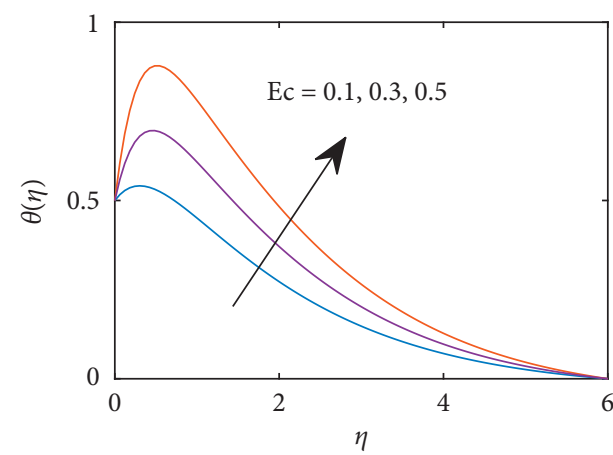

(b)

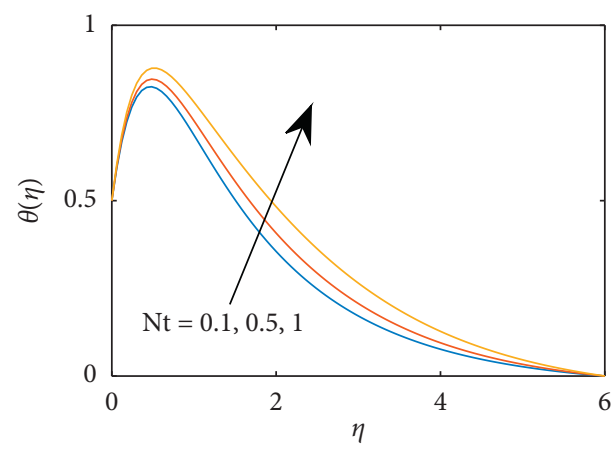

(d)

Figure 13: Impact of (a) $K$, (b) $E c$, (c) $N b$, (d) $N t$ on temperature profile. 


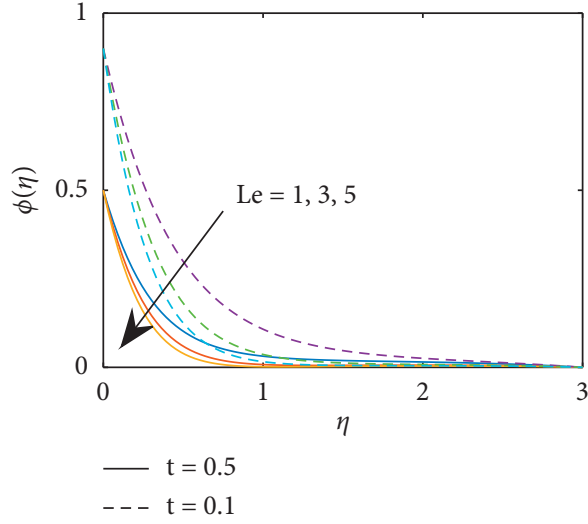

(a)

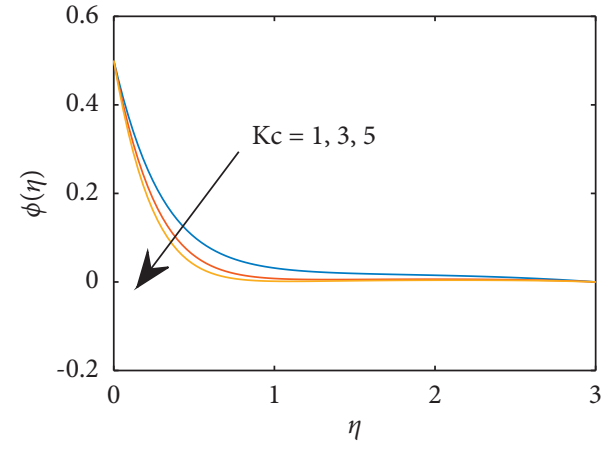

(b)

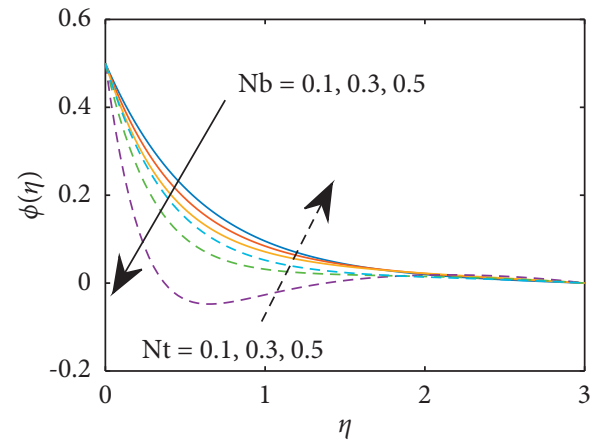

(c)

Figure 14: Impact of (a) Le, $t$ (b) $K c$, (c) $N b$, and $N t$ on concentration profile.

However increasing thermophoretic body force effect, $N t$, encourages the migration of nanoparticles under the temperature gradient towards colder regions in the boundary layer and this elevates nanoparticle concentration and therefore produces a greater solutal (nanoparticle) concentration boundary layer thickness.

Finally, Tables $1-3$ provide the results of the skin friction $\mathrm{C}_{f}$, wall couple stress (dimensionless microrotation gradient at the wall) $C_{s}$, local Nusselt number $N u_{x}$, and local Sherwood number, $S h_{x}$ ADM-Padé computations with respect to various contributing parameters. Table 1 shows the influence of micropolar material parameter, $K$, magnetic parameter, $M$, permeability parameter, $K p$, mixed convection parameter, $\lambda$, and buoyancy ratio parameter, $\delta$ on skin friction and couple stress coefficients. The skin friction (linear shear stress function) and the couple stress coefficient both increase as the material parameter increases. With increasing $M$ values (greater Lorentzian drag effect), the skin friction and couple stress coefficient are however suppressed. With increasing permeability effect $(K p)$, there is a decrease in Darcian porous impedance and the skin friction and wall couple stress are both enhanced. Elevation in mixed convection parameter $\lambda$ depletes skin friction and also couple stress coefficient, whereas an increment in the buoyancy ratio parameter $\delta$ produces the opposite effect; i.e., it increases the skin friction at the sheet and also boosts the couple stress coefficient. The local Nusselt number $N u_{x}$ quantifies the relative contribution of thermal convection to
TABLE 1: Skin friction and wall couple stress coefficient.

\begin{tabular}{lcccccc}
\hline$K$ & $M$ & $K p$ & $\lambda$ & $\delta$ & $C_{f}$ & $C_{s}$ \\
\hline 0 & 1 & 0.2 & 5 & 5 & -0.5973 & 0.4133 \\
0.1 & & & & & -0.649 & 0.4463 \\
0.1 & 2 & & & & 0.3638 & 0.2599 \\
& 3 & & & & 0.0827 & 0.0967 \\
& 1 & 0.3 & & & 1.25 & 0.8259 \\
& & 0.5 & & & 1.8019 & 1.218 \\
& & 0.2 & 2 & & -1.3116 & -0.6112 \\
& & & 3 & & -0.6418 & -0.2967 \\
& & & 5 & 3 & -0.2952 & -0.1214 \\
& & & & 4 & -0.7607 & 0.1564 \\
\hline
\end{tabular}

thermal conduction at the sheet surface and is tabulated for various parameters in Table 2. It is evident that increasing thermal radiation $(R d)$ and temperature ratio $(T r)$ parameters strongly enhances local Nusselt number; i.e., they produce a greater transfer of heat from the boundary layer to the wall. An increment in Eckert number $(E c)$ and the heat source parameter $(S)$ however suppresses the local Nusselt number $N u_{x}$ since these parameters heat the boundary layer and elevate temperatures. This will suppress the heat transferred to the wall and will decrease $N u_{x}$ magnitudes. Table 3 shows the variation of local Sherwood number $S h_{x}$ for different values of Brownian motion $(\mathrm{Nb})$, thermophoresis parameter $(N t)$, Lewis number $(L e)$, and chemical reaction parameter $(K c)$. Increment in Brownian motion 
TABLE 2: Nusselt number.

\begin{tabular}{lcccc}
\hline$R d$ & $T r$ & $E c$ & $S$ & $N u_{x}$ \\
\hline 2 & 2 & 0.5 & 0.01 & 0.3147 \\
3 & & & & 0.9333 \\
2 & 3 & & & 0.8827 \\
& 4 & 1 & & 1.381 \\
& 2 & 2 & & 0.2074 \\
& & 0.5 & 0.02 & -0.3889 \\
& & & 0.03 & 0.5117 \\
& & & & 0.4977 \\
\hline
\end{tabular}

TABLE 3: Sherwood number.

\begin{tabular}{ccccc}
\hline$N b$ & $N t$ & $K c$ & $L e$ & $S h_{x}$ \\
\hline 0.1 & 0.5 & 1 & 2 & 1.1994 \\
0.2 & & & & 1.1233 \\
0.5 & 0.1 & & & 1.0611 \\
& 0.2 & & & 1.0664 \\
& 0.5 & 5 & & 1.7908 \\
& & 1 & 1 & 2.287 \\
& & & 3 & 0.8116 \\
& & & & 1.2974 \\
\hline
\end{tabular}

parameter $(\mathrm{Nb})$ strongly reduces local Sherwood number, i.e., decreases the mass transfer of nanoparticles from the boundary layer stretching regime to the wall (sheet). The converse effect is computed for increasing thermophoresis parameter $(\mathrm{N} t)$, which elevates the local Sherwood number $S h_{x}$, i.e., results in greater migration of nanoparticles to the wall. Similarly, increments in Lewis number $(L e)$ and chemical reaction parameter $(K c)$ are both favourable for enhancing the rate of mass transfer of nanoparticle solute to the wall; i.e., stronger chemical reaction and higher thermal diffusivity produce greater diffusion of nanoparticles to the wall and a thinner concentration boundary layer thickness.

\section{Conclusions}

Inspired by more robust simulations of magnetic nonNewtonian nanofluid materials processing operations, a mathematical model has been developed for electrically conducting micropolar nanofluid flow from a vertical stretching surface adjacent to a porous medium under a transverse magnetic field. Eringen's micropolar model has been deployed for non-Newtonian characteristics and the Buongiorno nanofluid model employed for nanoscale effects (thermophoresis and Brownian motion). The model also includes double stratification (thermal and solutal) and also chemical reaction effects, heat source, and viscous dissipation. Darcy's model is employed for the porous medium and a Rosseland diffusion flux approximation for nonlinear thermal radiation. The nonlinear governing partial differential conservation equations are rendered into nonlinear ordinary differential equations via relevant transformations. An innovative semi-numerical methodology combining the Adomian decomposition method (ADM) with Padé approximants and known as ADM-Padé is deployed to solve the emerging non-linear ordinary differential boundary value problem with appropriate wall and freestream conditions in MATLAB software. A detailed parametric study of the influence of key parameters on stream function, velocity, microrotation (angular velocity), temperature, and nanoparticle concentration profiles is conducted. Furthermore, skin friction coefficient, wall couple stress coefficient, Nusselt number, and Sherwood number are displayed in tables. The validation of both numerical techniques used, i.e., ADM and ADMPadé, against a conventional numerical $4^{\text {th }}$-order Runge Kutta method is also included and significant acceleration in convergence of solutions achieved with the ADM-Padé approach. The main findings of the current study may be summarized as follows:

(i) The flow is decelerated with greater buoyancy ratio parameter whereas microrotation (angular velocity) is enhanced

(ii) Increasing thermal and solutal stratification suppresses microrotation

(iii) Concentration magnitudes are boosted with greater chemical reaction parameter and Lewis number

(iv) Temperatures are significantly enhanced with radiative parameter

(v) Increasing Brownian motion parameter depletes concentration values whereas increasing thermophoresis parameter enhances concentrations

(vi) Increases in Lewis number and chemical reaction parameter both enhance the local Sherwood number

(vii) An increment in Eckert number and the heat source parameter reduces local Nusselt number whereas they elevate temperatures and increase thermal boundary layer thickness

(viii) Greater magnetic parameter (stronger Lorentzian drag effect) reduces the skin friction and couple stress coefficient

(ix) With increasing permeability effect, there is a decrease in Darcian porous impedance and the skin friction and wall couple stress are both elevated

(x) Elevation in mixed convection parameter $\lambda$ reduces skin friction and also couple stress coefficient, whereas greater buoyancy ratio parameter increases the skin friction at the sheet and also boosts the couple stress coefficient

(xi) ADM-Padé method achieves faster convergence and improved accuracy relative to conventional $\mathrm{ADM}$ or Runge-Kutta numerical approaches

The present study finds applications in thermomagnetic coating processes involving nanomaterials with microstructural characteristics. Future studies may consider entropy generation minimization via the second law of thermodynamics. 


\section{Nomenclature}

$\begin{array}{ll}a: & \text { Stretching rate constant }\left[\mathrm{s}^{-1}\right] \\ t: & \text { Thermal stratification parameter } \\ T: & \text { Temperature of the fluid }[K] \\ b, c, d: & \text { Dimensionless constants } \\ T r: & \text { Temperature ratio parameter }[K] \\ B_{0}: & \text { Magnetic field strength } \\ T_{0}: & \text { Reference temperature }[K] \\ C: & \left.\text { Concentration field [Kgm }{ }^{-3}\right] \\ T_{w}: & \text { Temperature at wall surface }[K] \\ C_{0}: & \text { Reference concentration }\left[\mathrm{Kgm}^{-3}\right] \\ T_{\infty}: & \text { Ambient temperature }\left[K^{-3}\right] \\ C_{w}: & \text { Concentration at the wall of the surface } \\ U_{w}: & \text { Velocity of the surface }\left[\mathrm{ms}^{-1}\right] \\ C_{\infty}: & \text { Ambient concentration }\left[\mathrm{Kgm}^{-3}\right] \\ u, v: & \text { Fluid velocities along } x, y \text { axes }\left[\mathrm{ms}^{-1}\right] \\ D_{T}: & \text { Thermophoretic diffusion coefficient } \\ D_{B}: & \text { Brownian diffusion coefficient } \\ E c: & \text { Eckert number } \\ x, y: & \text { Coordinate axes along and perpendicular to the } \\ & \text { surface } \\ g: & \text { Dimensionless stream function } \\ g: & \text { Gravitational acceleration }\left[\mathrm{ms}^{-2}\right] \\ G r: & \text { Thermal Grashof number. }\end{array}$

\section{Greek Symbols}

j: $\quad$ Micro inertia density

$\phi:$ Dimensionless concentration $\left[\mathrm{Kgm}^{-3}\right]$

$k$ : Vortex viscosity

$\omega$ : Dimensionless stream function

$K$ : Micropolar material parameter

$\rho_{f}:$ Density of fluid $\left[\mathrm{Kgm}^{-3}\right]$

$k^{*}$ : Mean absorption coefficient

$\rho_{p}:$ Density of nanoparticles $\left[\mathrm{Kgm}^{-3}\right]$

$K c$ : Chemical reaction parameter

$\sigma: \quad$ Electrical conductivity $\left[\Omega^{-1} \mathrm{~m}^{-1}\right]$

$K p$ : Porosity parameter

$\sigma^{*}$ : Stefan-Boltzmann constant

$k_{p}^{*}$ : Permeability of porous medium

$\beta_{c}$ : Volumetric coefficient of solutal expansion

$K_{r}^{*}$ : Reaction rate constant

Le: Lewis number

$\beta_{T}$ : Volumetric coefficient of thermal expansion

$M$ : Magnetic parameter

$m$ : Microgyration parameter

$\mu: \quad$ Dynamic viscosity $\left[\mathrm{Kgm}^{-1} \mathrm{~s}^{-1}\right]$

$N$ : Angular velocity component $\left[\mathrm{ms}^{-1}\right]$

$\alpha_{f}$ : Thermal diffusivity $\left[\mathrm{m}^{2} \mathrm{~s}^{-1}\right]$

$N b$ : Brownian motion parameter

$\eta$ : Similarity variable

$N t$ : Thermophoresis parameter

$\theta: \quad$ Dimensionless temperature $[K]$

Pr: Prandtl number

$\lambda$ : Mixed convection parameter

$Q: \quad$ Heat source

$\delta$ : Buoyancy ratio parameter

$q_{r}$ : Radiative heat flux $\gamma: \quad$ Spin gradient viscosity

$R d$ : Radiation parameter

$\tau$ : $\quad$ Relative heat capacity

Re: Local Reynolds number

$S$ : Dimensionless heat source.

\section{Subscripts}

$s$ : Solutal stratification parameter

$f$ : Base fluid.

\section{Data Availability}

No data were used to support this study.

\section{Conflicts of Interest}

The authors declare that they have no conflicts of interest.

\section{References}

[1] B. C. Sakiadis, "Boundary-layer behavior on continuous solid surfaces: I. Boundary-layer equations for two-dimensional and axisymmetric flow," AIChE Journal, vol. 7, no. 1, pp. 26-28, 1961.

[2] B. C. Sakiadis, "Boundary-layer behavior on continuous solid surfaces: II. The boundary layer on a continuous flat surface," AIChE Journal, vol. 7, no. 2, pp. 221-225, 1961.

[3] L. J. Crane, "Flow past a stretching plate," Zeitschrift für angewandte Mathematik und Physik ZAMP, vol. 21, no. 4, pp. 645-647, 1970.

[4] S. R. Mishra, P. K. Pattnaik, M. M. Bhatti, and T. Abbas, "Analysis of heat and mass transfer with MHD and chemical reaction effects on viscoelastic fluid over a stretching sheet," Indian Journal of Physics, vol. 91, no. 10, pp. 1219-1227, 2017.

[5] A. J. Chamkha, A. M. Rashad, and H. F. Al-Mudhaf, "Heat and mass transfer from truncated cones with variable wall temperature and concentration in the presence of chemical reaction effects," International Journal of Numerical Methods for Heat and Fluid Flow, vol. 22, no. 3, pp. 357-376, 2012.

[6] B. Mallikarjuna, A. M. Rashad, A. J. Chamkha, and S. H. Raju, "Chemical reaction effects on MHD convective heat and mass transfer flow past a rotating vertical cone embedded in a variable porosity regime," Afrika Matematika, vol. 27, no. 3, pp. 645-665, 2016.

[7] K. A. Khan, A. R. Butt, N. Raza, and K. Maqbool, "Unsteady magneto-hydrodynamics flow between two orthogonal moving porous plates," The European Physical Journal Plus, vol. 134, no. 1, p. 1, 2019.

[8] K. A. Khan, A. R. Butt, and N. Raza, "Influence of porous medium on magneto hydrodynamics boundary layer flow through elastic sheet with heat and mass transfer," Journal of Nanofluids, vol. 8, no. 4, pp. 725-735, 2019.

[9] O. D. Makinde, "Heat and mass transfer by MHD mixed convection stagnation point flow toward a vertical plate embedded in a highly porous medium with radiation and internal heat generation," Meccanica, vol. 47, no. 5, pp. 1173-1184, 2012.

[10] J. R. Pattnaik, G. C. Dash, and S. Singh, "Radiation and mass transfer effects on MHD flow through porous medium past an exponentially accelerated inclined plate with variable temperature," Ain Shams Engineering Journal, vol. 8, no. 1, pp. 67-75, 2017. 
[11] J. R. Pattnaik, G. C. Dash, and S. Singh, "Diffusion-thermo effect with Hall current on unsteady hydromagnetic flow past an infinite vertical porous plate," Alexandria Engineering Journal, vol. 56, no. 1, pp. 13-25, 2017.

[12] R. Kandasamy, K. Periasamy, and K. K. S. Prabhu, "Effects of chemical reaction, heat and mass transfer along a wedge with heat source and concentration in the presence of suction or injection," International Journal of Heat and Mass Transfer, vol. 48, no. 7, pp. 1388-1394, 2005.

[13] A. C. Eringen, "Simple microfluids," International Journal of Engineering Science, vol. 2, no. 2, pp. 205-217, 1964.

[14] A. Eringen, "Theory of micropolar fluids," Indiana University Mathematics Journal, vol. 16, no. 1, pp. 1-18, 1966.

[15] A. C. Eringen, "Theory of thermomicrofluids," Journal of Mathematical Analysis and Applications, vol. 38, no. 2, pp. 480-496, 1972.

[16] R. Bhargava and H. S. Takhar, "Numerical study of heat transfer characteristics of the micropolar boundary layer near a stagnation point on a moving wall," International Journal of Engineering Science, vol. 38, no. 4, pp. 383-394, 2000.

[17] M. Ezzat, A. A. El-Bary, and S. Ezzat, "Combined heat and mass transfer for unsteady MHD flow of perfect conducting micropolar fluid with thermal relaxation," Energy Conversion and Management, vol. 52, no. 2, pp. 934-945, 2011.

[18] E. P. Douglas, "Processing of polymers using magnetic fields," in Proceedings of the International Workshop on Materials Analysis and Processing in Magnetic Fields, pp. 17-19, Tallahassee, FL, USA, March 2004.

[19] S. R. Mishra, P. K. Pattnaik, and G. C. Dash, "Effect of heat source and double stratification on MHD free convection in a micropolar fluid," Alexandria Engineering Journal, vol. 54, no. 3, pp. 681-689, 2015.

[20] S. U. Choi and J. A. Eastman, "Enhancing thermal conductivity of fluids with nanoparticles," Argonne National Lab., Argonne, IL, USA, No. ANL/MSD/CP-84938; CONF-95113529, Argonne, IL, USA, 1995.

[21] A. Wakif, Z. Boulahia, S. R. Mishra, M. Mehdi Rashidi, and R. Sehaqui, "Influence of a uniform transverse magnetic field on the thermo-hydrodynamic stability in water-based nanofluids with metallic nanoparticles using the generalized Buongiorno's mathematical model," The European Physical Journal Plus, vol. 133, no. 5, p. 181, 2018.

[22] T. Hayat, A. Aziz, T. Muhammad, and B. Ahmad, "Influence of magnetic field in three-dimensional flow of couple stress nanofluid over a nonlinearly stretching surface with convective condition," PLoS One, vol. 10, no. 12, p. e0145332, 2015.

[23] M. M. Bhatti, M. A. Yousif, S. R. Mishra, and A. Shahid, "Simultaneous influence of thermo-diffusion and diffusionthermo on non-Newtonian hyperbolic tangent magnetised nanofluid with Hall current through a nonlinear stretching surface," Pramana, vol. 93, no. 6, pp. 1-10, 2019.

[24] M. Khan, L. Ahmad, and W. A. Khan, "Numerically framing the impact of radiation on magnetonanoparticles for 3D Sisko fluid flow," Journal of the Brazilian Society of Mechanical Sciences and Engineering, vol. 39, no. 11, pp. 4475-4487, 2017.

[25] M. Modest, Radiative Heat Transfer, MacGraw-Hill, New York, NY, USA, 1993

[26] G. Adomian, "Solving frontier problems of physics: the decomposition method, with a preface by Yves Cherruault," in Fundamental Theories of PhysicsVol. 1, Kluwer Academic Publishers Group, Dordrecht, The Netherlands, 1994.
[27] G. Adomian, Nonlinear Stochastic Systems Theory and Applications to Physics, Vol. 46, Springer Science \& Business Media, Berlin, Germany, 1988.

[28] G. A. Baker and P. R. Graves-Morris, Padé Approximants, Cambridge University Press, Cambridge, UK, 2nd edition, 1996.

[29] M. B. Arain, M. M. Bhatti, A. Zeeshan, and F. S. Alzahrani, "Bioconvection Reiner-Rivlin nanofluid flow between rotating circular plates with induced magnetic effects, activation energy and squeezing phenomena," Mathematics, vol. 9, no. 17, p. 2139, 2021.

[30] M. Ramzan, N. Ullah, J. D. Chung, D. Lu, and U. Farooq, "Buoyancy effects on the radiative magneto Micropolar nanofluid flow with double stratification, activation energy and binary chemical reaction," Scientific Reports, vol. 7, no. 1, pp. 12901-12915, 2017. 